This is the accepted manuscript made available via CHORUS. The article has been published as:

\title{
Matter imprints in waveform models for neutron star binaries: Tidal and self-spin effects
}

Tim Dietrich, Sebastian Khan, Reetika Dudi, Shasvath J. Kapadia, Prayush Kumar, Alessandro Nagar, Frank Ohme, Francesco Pannarale, Anuradha Samajdar, Sebastiano

Bernuzzi, Gregorio Carullo, Walter Del Pozzo, Maria Haney, Charalampos Markakis, Michael Pürrer, Gunnar Riemenschneider, Yoshinta Eka Setyawati, Ka Wa Tsang, and Chris Van Den Broeck

Phys. Rev. D 99, 024029 - Published 18 January 2019

DOI: 10.1103/PhysRevD.99.024029 


\title{
Matter imprints in waveform models for neutron star binaries: tidal and self-spin effects
}

\author{
Tim Dietrich ${ }^{1,2}$, Sebastian Khan ${ }^{3,4}$, Reetika Dudi ${ }^{5}$, Shasvath J. Kapadia ${ }^{6}$, Prayush Kumar ${ }^{7}$, Alessandro \\ Nagar $^{8,9,10}$, Frank Ohme ${ }^{3,4}$, Francesco Pannarale ${ }^{11,12}$, Anuradha Samajdar ${ }^{1}$, Sebastiano Bernuzzi ${ }^{5,13,14}$, \\ Gregorio Carullo $^{15}$, Walter Del Pozzo ${ }^{15}$, Maria Haney ${ }^{16}$, Charalampos Markakis ${ }^{17,18}$, Michael Pürrer ${ }^{2}$, \\ Gunnar Riemenschneider ${ }^{9,19}$, Yoshinta Eka Setyawati ${ }^{3,4}$, Ka Wa Tsang ${ }^{1}$, and Chris Van Den Broeck ${ }^{1,20}$ \\ 1 Nikhef, Science Park, 1098 XG Amsterdam, The Netherlands \\ ${ }^{2}$ Max Planck Institute for Gravitational Physics (Albert Einstein Institute), Am Mühlenberg 1, Potsdam 14476, Germany \\ ${ }^{3}$ Max Planck Institute for Gravitational Physics (Albert Einstein Institute), Callinstr. 38, 30167 Hannover, Germany \\ 4 Leibniz Universität Hannover, D-30167 Hannover, Germany \\ 5 Theoretical Physics Institute, University of Jena, 07743 Jena, Germany \\ ${ }^{6}$ Center for Gravitation, Cosmology, and Astrophysics, \\ University of Wisconsin-Milwaukee, Milwaukee, Wisconsin 53201, USA \\ 7 Cornell Center for Astrophysics and Planetary Science, \\ Cornell University, Ithaca, New York 14853, USA \\ 8 Centro Fermi - Museo Storico della Fisica e Centro Studi e Ricerche Enrico Fermi, Rome, Italy \\ ${ }^{9}$ INFN Sezione di Torino, Via P. Giuria 1, 10125 Torino, Italy \\ 10 Institut des Hautes Etudes Scientifiques, 91440 Bures-sur-Yvette, France \\ 11 Gravity Exploration Institute, School of Physics and Astronomy, \\ Cardiff University, The Parade, Cardiff CF24 3AA, UK \\ 12 Dipartimento di Fisica, Università di Roma "Sapienza" ES Sezione INFN Roma1, P.A. Moro 5, 00185, Roma, Italy \\ 13 Dipartimento di Scienze Matematiche Fisiche ed Informatiche, Universitá di Parma, I-43124 Parma, Italia \\ 14 Istituto Nazionale di Fisica Nucleare, Sezione Milano Bicocca, \\ gruppo collegato di Parma, I-43124 Parma, Italia \\ 15 Dipartimento di Fisica Enrico Fermi, Universit di Pisa, Pisa I-56127, Italy \\ 16 Physik-Institut, Universität Zürich, Winterthurerstrasse 190, 8057 Zürich, Switzerland \\ 17 NCSA, University of Illinois at Urbana-Champaign, 1205 W Clark St, Urbana, Ilinois 61801, USA \\ 18 DAMTP, Centre for Mathematical Sciences, Wilberforce Road, \\ University of Cambridge, Cambridge, CB3 0WA, UK \\ 19 Dipartimento di Fisica, Universit' 'a di Torino, via P. Giuria 1, I-10125 Torino, Italy and \\ 20 Van Swinderen Institute for Particle Physics and Gravity, \\ University of Groningen, Nijenborgh 4, 9747 AG Groningen, The Netherlands
}

\begin{abstract}
The combined observation of gravitational and electromagnetic waves from the coalescence of two neutron stars marks the beginning of multi-messenger astronomy with gravitational waves (GWs). The development of accurate gravitational waveform models is a crucial prerequisite to extract information about the properties of the binary system that generated a detected GW signal. In binary neutron star systems (BNS), tidal effects also need to be incorporated in the modeling for an accurate waveform representation. Building on previous work [Phys. Rev. D96 121501], we explore the performance of inspiral-merger waveform models that are obtained by adding a numerical relativity (NR) based approximant for the tidal part of the phasing (NRTidal) to existing models for nonprecessing and precessing binary black hole systems, as implemented in the LSC Algorithm Library Suite. The resulting BNS waveforms are compared and contrasted to a set of target waveforms which we obtain by hybridizing NR waveforms (covering the last $\sim 10$ orbits up to merger and extending through the postmerger phase) with inspiral waveforms calculated from $30 \mathrm{~Hz}$ obtained with a state-of-the-art effective-one-body waveform model. While due to the construction procedure of the target waveforms, there is no error budget available over the full frequency range accessible by advanced GW detectors, the waveform set presents only an approximation of the real signal. We probe that the combination of the self-spin terms and of the NRTidal description is necessary to obtain minimal mismatches $(\lesssim 0.01)$ and phase differences $(\lesssim 1 \mathrm{rad})$ with respect to the target waveforms. We also discuss possible improvements and drawbacks of the NRTidal approximant in its current form.
\end{abstract}

\section{INTRODUCTION}

On August 17, 2017, the gravitational wave (GW) detector network formed by the Advanced LIGO and Virgo interferometers detected GW170817, the first GW signal consistent with the inspiral and merger of a binary neutron star (BNS) system [1]. In addition to the GW signal, astronomers observed the short gamma-ray burst GRB 170817A 2] and the transient AT 2017gfo in the Xray, ultraviolet, optical, infrared, and radio bands [3, 4] from the same source. This joint detection initiated a new era of multi-messenger astronomy. From this single observation it was already possible to prove that BNS mergers are central engines for short gamma-ray bursts, 
and that they produce heavy elements which give rise to electromagnetic counterparts known as kilonovae or macronovae. Additionally, measurements of the speed of GWs [2] as well as of the Hubble constant were performed [5]. Finally, new constraints on the unknown equation-of-state (EOS) of cold matter at supranuclear densities were determined, e.g. [1, 6, 17. Due to the increasing sensitivity of advanced GW detectors over the next years, multiple detections of merging BNSs are expected in the near future [8].

Extracting information about the properties of the binary system from GW detector data is crucial for the field of GW astronomy. Source properties are generally inferred via a coherent, Bayesian analysis that involves repeated cross-correlation of the measured GW strain with predicted waveforms [9. Therefore, the computation of individual waveforms needs to be efficient and fast. Furthermore, in contrast to binary black hole (BBH) systems, which are usually detectable for the last few orbits before merger, BNS systems are visible by GW detectors for several seconds or even minutes before the merger. Consequently, computational efficiency is even more important for BNS waveform approximants than for BBH systems. On the other hand, the computed waveforms need to be an accurate representation of the binary system to allow for correct estimates of the source properties, such as the masses and spins, and, in the case of BNS systems, the internal structure of the stars.

Significant progress in modeling BNS systems was accomplished over the last few years, capturing the stronggravity and tidally dominated regime of the late-inspiral. State-of-the-art tidal waveform models in the time domain have been developed in [10 12] and are based on the effective-one-body (EOB) description of the generalrelativistic two-body problem [13, 14]. While this approach is powerful and accurately describes the waveform up to the moment of merger for a variety of binary configurations within the uncertainty of state-ofthe-art numerical relativity (NR) simulations, there are BNS parameter space regions for which recent numerical simulations suggest that further improvements of the tidal EOB models are necessary [15, 16. But, the biggest disadvantage of this approach is the high computational cost required to compute a single waveform. While applying reduced-order-modeling techniques [17. allows to overcome this issue, it also adds additional complexity. Therefore, modeling techniques complementary to EOB, e.g. [18 21], are needed, especially because PostNewtonian (PN) approximants become increasingly inaccurate towards the merger, e.g. 22 25].

In Ref. [26, the authors propose the first closed-form tidal approximant combining PN, tidal EOB, and NR information. This waveform model was implemented in the LSC Algorithm Library (LAL) Suite 27. to support the analysis of GW170817 [1. Specifically, a particular version of this tidal approximant was added to the pointmass dynamics described by the spin-aligned EOB model of [28] and by the phenomenological, frequency-domain approach of [29 31. Very recently [32] also developed a tidal approximant in the frequency domain combining EOB and NR information, for a comparison between the NRTidal model and the model of [32] we refer the reader to Appendix $\mathrm{E}$ of 32 .

In addition to the tidal interaction, another EOSdependent effect that distinguishes BNSs from $\mathrm{BBH}$ binaries is the deformation that the star acquires due to its own rotation (self-spin or monopole-quadrupole terms), that eventually leaves an imprint on the gravitationalwave signal [33. The outcome of the leading-order (LO) PN-based description of this effect on EOS measurements has been investigated in recent works [34 36] and it has been incorporated, in resummed form, in the TEOBResumS EOB model [37 and in the SEOBNRv4T 11, 12 model.

The main goal of this paper is to assess the quality of the implementation of tidal effects described by the NRTidal model in the LALSuite as well as of the PN-description of EOS-dependent self-spin effects. This is done by comparing LALSuite BNS waveforms to hybrid waveforms obtained by matching together NR waveforms, covering the last $\sim 10$ orbits up to merger and extending through the postmerger phase, with inspiral waveforms (calculated from $30 \mathrm{~Hz}$ ) obtained with TEOBResumS. We note that since there is no error budget for the hybrid waveforms available throughout the entire frequency range accessible by advanced GW detectors, the target waveforms should only be interpreted as an approximation of the true signal. Thus, final presented results could lead in some scenarios to a biased estimate.

In Sec. II, we discuss the tidal phase correction which is the key element of transforming a BBH baseline model to obtain BNS waveforms, as well as the BBH baseline models we employed. We discuss the NR simulations and hybrids used for our tests in Sec. III] and validate the models in the frequency domain and the time domain in Sec. IV and in Sec. V] respectively, using either mismatches or direct phase comparisons. Finally, Sec. VI points out systematic effects that are present in the current implementation of NRTidal that may affect parameter estimation studies introducing biases. Our conclusions are collected in Sec. VII.

Throughout this article geometric units are used by setting $G=c=M_{\odot}=1$.

\section{IMR_NRTIDAL MODELS}

\section{A. Model description}

\section{Numerical relativity and effective-one-body tuned tidal phase correction}

In contrast to the $\mathrm{BBH}$ case, waveforms describing the emission from BNS systems need to include tidal effects that incorporate the fact that each star gets tidally polarized due to the tidal field of the companion [38 41]. 
In the following, we include tidal effects by means of the method outlined in [26], where the tidal phase has been extracted from the tidal EOB model of [10 and high-resolution BNS NR waveforms. The procedure is outlined here; the interested reader can find a detailed discussion in Ref. [26].

We consider a binary with total mass $M=M_{A}+M_{B}$, with the convention that $M_{A} \geq M_{B}$. Defining the complex GW as $h(t)=A(t) e^{-\mathrm{i} \phi(t)}$, the time-domain phase $\phi(t)$ is assumed to be given by the following $\mathrm{PN}$-inspired sum of individual contributions

$$
\phi(\hat{\omega}) \approx \phi_{\mathrm{pp}}(\hat{\omega})+\phi_{\mathrm{spin}}(\hat{\omega})+\phi_{\mathrm{tides}}(\hat{\omega})
$$

where $\hat{\omega}=M \omega=M \partial_{t} \phi(t)$ is the dimensionless GW frequency, $\phi_{\mathrm{pp}}(\hat{\omega})$ denotes the nonspinning, point-particle, contribution to the overall phase, $\phi_{\text {spin }}$ corresponds to contributions caused by spin effects, and $\phi_{\text {tides }}$ corresponds to contributions caused by tidal effects. As shown in [26, 42] one finds that during the last orbits before merger, i.e., the regime accessible by NR simulations, and for dimensionless spin magnitudes up to $S_{A, B} / M_{A, B}^{2} \sim 0.1$ current state-of-the-art NR simulations are not capable of revealing tides-spin coupling, which supports the specific form of Eq. (1) and the absence of a $\phi_{\text {spin } \leftrightarrow \text { tides }}$ term.

Non-spinning tidal contributions enter the phasing at the 5PN order 1 . The fully known next-to-leading order (NLO) PN expression of the tidal contribution (TaylorT2 approximant) [14, 24, 45, 46] is

$$
\phi_{\mathrm{tides}}=-\kappa_{\mathrm{eff}}^{T} \frac{c_{\mathrm{Newt}} x^{5 / 2}}{X_{A} X_{B}}\left(1+c_{1} x\right),
$$

with $x(\hat{\omega})=(\hat{\omega} / 2)^{2 / 3}, c_{\text {Newt }}=-13 / 8$ and $X_{A, B}=$ $M_{A, B} / M$. The NLO tidal correction to the phasing, $c_{1}$, is for the equal-mass case $c_{1}=1817 / 364$.

The parameter $\kappa_{\text {eff }}^{T}$ characterized tidal effects and reads

$$
\kappa_{\mathrm{eff}}^{T}=\frac{2}{13}\left[\left(1+12 \frac{X_{B}}{X_{A}}\right)\left(\frac{X_{A}}{C_{A}}\right)^{5} k_{2}^{A}+(A \leftrightarrow B)\right],
$$

where $C_{A, B} \equiv M_{A, B} / R_{A, B}$ are the compactnesses of the stars at isolation, and $k_{2}^{A, B}$ the Love numbers describing the static quadrupolar deformation of one body in the gravitoelectric field of the companion [40. The tidal parameter $\kappa_{\text {eff }}^{T}$ is connected to $\tilde{\Lambda}$ used to characterize tidal effects in Ref. [1] via $\tilde{\Lambda}=16 / 3 \kappa_{\text {eff }}^{T}$, once one has defined the tidal polarizability parameters as $\Lambda_{A, B}=2 / 3 k_{2}^{A, B} / C_{A, B}^{5}$.

\footnotetext{
1 As mentioned in the introduction, EOS dependent phase corrections depending on the self-spin interaction, the quadrupolemonopole terms, appear already at 2PN [43] (see also Ref. 44] for the NLO contributions). As shown in Sec. IV] and discussed, e.g. in [36], those effects are important for spinning BNS systems even with dimensionless spins $\sim 0.1$.
}

An effective representation of the tidal effects coming beyond the NLO Eq. (2) can be obtained using the following expression

$$
\phi_{\text {tides }}=-\kappa_{\text {eff }}^{T} \frac{c_{\text {Newt }} x^{5 / 2}}{X_{A} X_{B}} P_{\phi}^{\text {NRTidal }}(\hat{\omega}),
$$

where $P_{\phi}^{\mathrm{NRTidal}}(\hat{\omega})$ is fitted to $\mathrm{PN}, \mathrm{EOB}$, and NR waveforms in such a way that for $\hat{\omega} \leq 0.0074$ Eq. (2) is used to determine $P_{\phi}(\hat{\omega})$, for $\hat{\omega} \in[0.0074,0.04]$ the tidal EOB waveforms of [10, 47, 48] are used, and, finally, Richardson-extrapolated NR data ${ }^{2}$ of [26] are used for $\hat{\omega} \in[0.04,0.17]$, cf. Fig. 11. The final expression for $P_{\phi}(\hat{\omega})$ is represented by a rational function of the form

$$
P_{\phi}^{\text {NRTidal }}(\hat{\omega})=\frac{1+n_{1} x+n_{3 / 2} x^{3 / 2}+n_{2} x^{2}+n_{5 / 2} x^{5 / 2}+n_{3} x^{3}}{1+d_{1} x+d_{3 / 2} x^{3 / 2}} .
$$

We require that Eq. (5) reproduces Eq. (2) at low frequencies which is ensured when $d_{1}=\left(n_{1}-c_{1}\right)$. The coefficients are given by $\left(n_{1}, n_{3 / 2}, n_{2}, n_{5 / 2}, n_{3}\right)=$ $(-17.941,57.983,-298.876,964.192,-936.844), \quad$ and $d_{3 / 2}=43.446$.

The tidal phase correction in the frequency domain is computed from the time-domain approximant via stationary phase approximation 49. The integration is performed numerically and the numerical data are represented as

$$
\Psi^{\text {NRTidal }}(f)=-\kappa_{\text {eff }}^{T} \frac{\tilde{c}_{\text {Newt }}}{X_{A} X_{B}} x^{5 / 2} P_{\Psi}^{\text {NRTidal }},
$$

with

$$
P_{\Psi}^{\mathrm{NRTidal}}=\frac{1+\tilde{n}_{1} x+\tilde{n}_{3 / 2} x^{3 / 2}+\tilde{n}_{2} x^{2}+\tilde{n}_{5 / 2} x^{5 / 2}}{1+\tilde{d}_{1} x+\tilde{d}_{3 / 2} x^{3 / 2}},
$$

where $x=x(f), \tilde{c}_{\text {Newt }}=39 / 16$ and $\tilde{d}_{1}=\tilde{n}_{1}-$ $3115 / 1248$, while the remaining parameters are determined by fitting. They $\operatorname{read}\left(\tilde{n}_{1}, \tilde{n}_{3 / 2}, \tilde{n}_{2}, \tilde{n}_{5 / 2}\right)=$ $(-17.428,31.867,-26.414,62.362)$ and $\tilde{d}_{3 / 2}=36.089$. Similarly to Eq. (5), $\tilde{d}_{1}=\left(\tilde{n}_{1}-\tilde{c}_{1}\right)$ ensures that the NLO tidal term is correctly recovered.

Equation (6) gives then the final NRTidal correction which can be added to any tidal-free waveform model. We will discuss all current point-mass baseline models to which the NRTidal correction has been added in the next subsection. A comparison between $\Psi^{\mathrm{NRTidal}}$, Eq. (6), and PN tidal predictions is shown in Fig. 1. We show $\Psi_{\text {tides }} / \kappa_{\text {eff }}^{T}$ on a linear, semi-logarithmic, and double logarithmic scale. Additionally, we mark the intervals for which we used PN, EOB, NR datapoints to tune the NRTidal model. As seen in the bottom panel of Fig. 1 .

\footnotetext{
2 For calibration of the NRTidal model to NR data the configurations $\mathrm{SLy}_{1.35 \mid 1.35}^{0.00 \mid 0.00}, \mathrm{H} 4_{1.37 \mid 1.37}^{0.00 \mid 0.00}$, and $\mathrm{MS}_{1.35 \mid 1.35}^{0.00 \mid 0.00}$ have been used, cf. 26] for a detailed discussion.
} 


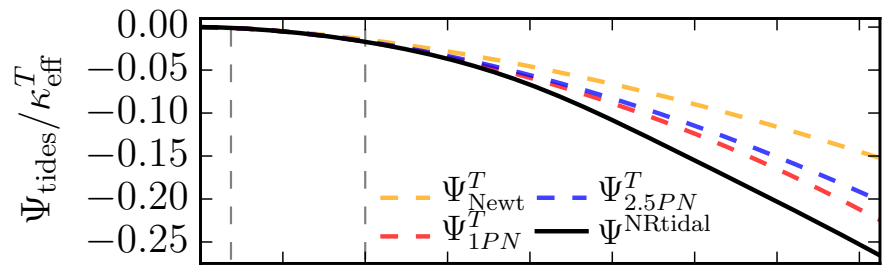

0.000 .020 .040 .060 .080 .100 .120 .140 .16
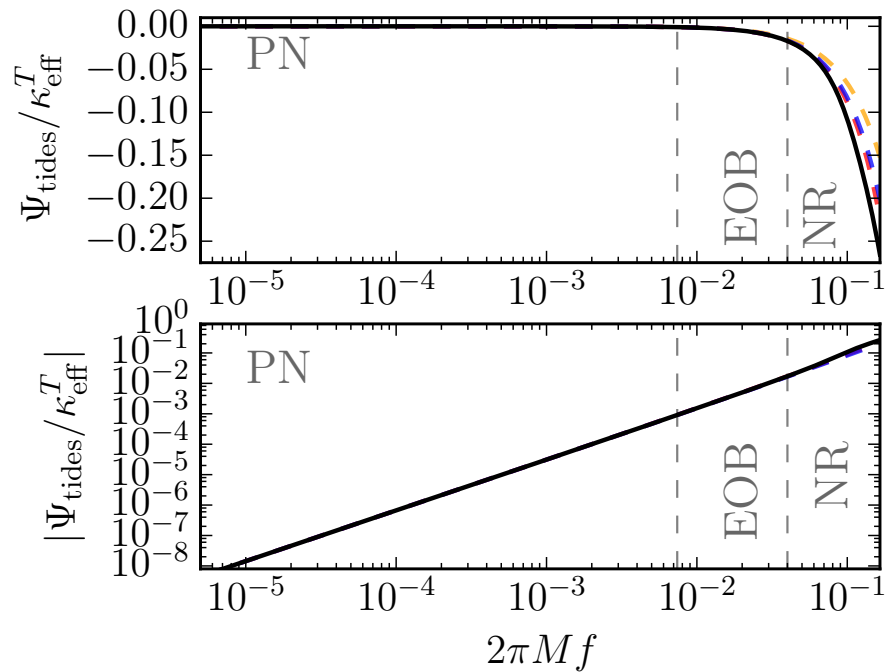

FIG. 1. Tidal phase correction for the NRTidal (black) and various $\mathrm{PN}$ models truncated at the corresponding $\mathrm{PN}$ orders from Eq. 20) (for comparison we restrict the PN models to the $\ell=2$ tidal component). From top to bottom, the phase correction is shown on a linear, on a semi-logarithmic, and on a double logarithmic scale, respectively. The vertical, dashed lines mark regions in which we calibrate the NRTidal model to $\mathrm{PN}, \mathrm{EOB}$, and NR waveforms.

only the last part of the inspiral is affected by the calibration to EOB and NR waveforms.

In this respect, we want to stress that the choice of explicitly incorporating analytical NLO tidal information in the above formulas was made mainly for simplicity and to reduce the number of parameters in Eq. (5). In fact, tidal information beyond NLO are available 49, see the discussion in Sec. VIA.

\section{Point-particle baseline models}

Models for the GW signal from BBH systems are under active development for use in the analysis of Advanced LIGO-Virgo data. Typically, the complex GW strain $h$ is decomposed into a spin-weight -2 spherical harmonic basis, i.e.,

$$
h(t ; \theta, \phi)=\sum_{\ell \geqslant 2} \sum_{m=-\ell}^{\ell} h_{\ell, m}(t) Y_{\ell, m}^{-2}(\theta, \phi),
$$

where for comparable mass, non-precessing systems the $\ell=|m|=2$ multipoles are dominant.
Two of the models used in the analysis of Advanced LIGO-Virgo data are the EOB model SEOBNRv4 28 and the phenomenological model PhenomD 29, 30. These are aligned-spin models for the $\ell=|m|=2$ multipoles that use PN/EOB to describe the early inspiral and then calibrate model coefficients to NR waveforms to predict the late-inspiral, merger, and ringdown. The ringdown portion of the models also incorporate information from black hole perturbation theory in the form of complex quasi-normal mode frequencies.

The agreement between these aligned-spin BBH models was quantified in 28]. With increasing mass ratio and for positively aligned spins with a magnitude above $\sim 0.5$, their agreement drops and their mismatch exceeds $3 \%$. However, the two BBH models agree in large regions of the BNS parameter space, and we therefore expect negligible differences between the models when we compare them against the set of NR waveforms we use in this study.

Contrary to the aligned-spin waveform models, PhenomPv2 includes precession effects. It is built upon the assumption that the spin-orbit coupling can be approximately separated into components parallel and perpendicular to the instantaneous orbital angular momentum, with the former influencing the rate of inspiral and the latter driving the precessional motion [50 55].

\section{B. LALSuite implementation}

\section{Addition of tidal phase}

The simplicity of the tidal correction given via Eq. (6) allows us to add $\Psi^{\text {NRTidal }}$ to any frequency domain waveform model which accurately represents the pointparticle or $\mathrm{BBH}$ coalescence. We construct tidal models of the SEOBNRv4 and PhenomD models called SEOBNRv4_ROM_NRTidal and PhenomD_NRTidal, respectively.

The construction permits a particularly simple implementation where we add the $\Psi^{\text {NRTidal }}$ correction along with a suitable amplitude $A^{\text {NRTidal }}$ to the point-particle GW polarizations obtained from the LALSimulation library according to the following equation

$$
h^{\text {tidal }}=h^{\mathrm{pp}} \cdot\left(A^{\mathrm{NRTidal}} e^{-i \Psi^{\mathrm{NRTidal}}}\right),
$$

where $A^{\text {NRTidal }}$ is a function that smoothly turns off the waveform shortly after the termination frequency described below.

To construct a precessing tidal waveform approximant from the PhenomPv2 BBH baseline model, we add the tidal correction to the underlying spin-aligned PhenomD model on which PhenomPv2 is built on before we rotate the waveform according to the angles that describe the precession dynamics [54, [55]. At leading order, the tidal effects decouple from the precessional motion and the resulting precessing waveform model should still be valid. 
The inspiral part of PhenomPv2 (and PhenomD) is based upon the TaylorF2 approximant. This allows us to include the NLO effect due to the spin-induced quadrupolemonopole interaction [33, which currently is only included in the PhenomPv2_NRTidal mode 3 . This modifies the spin contribution to the quadrupole moment, which is a function of the EOS. Here we utilize the universal relations of 56 to relate the tidal deformability parameters $\Lambda_{1,2}$ to the spin-induced quadrupole-monopole terms. These terms in the PhenomPv2_NRTidal waveform model occur at the NLO in the inspiral phase. Note that the universal relations do not incorporate the black hole limit for $\Lambda \rightarrow 0$, thus we do propose to restrict their usage only above $\Lambda>1$ for which very good agreement is obtained. For the configurations considered in this work the difference between employing/not-employing universal relations is about 2-3 orders of magnitude smaller than the accumulated tidal phase.

\section{Termination criterion for tidal correction}

The tidal phase correction in Eq. (6) only describes the inspiral part of the BNS coalescence. Therefore, an additional criterion where to stop the computation of the waveform is required. We relate this termination criterion to the merger frequency. As outlined in [57, (for moderate mass ratios) the merger frequency of BNS systems is a function of

$$
\kappa_{2}^{T}=2\left[\frac{X_{B}}{X_{A}}\left(\frac{X_{A}}{C_{A}}\right)^{5} k_{2}^{A}+\frac{X_{A}}{X_{B}}\left(\frac{X_{B}}{C_{B}}\right)^{5} k_{2}^{B}\right] .
$$

Note that in the LAL implementation $\kappa_{2}^{T}$ is substituted by $\kappa_{\text {eff }}^{T}$ for simplicity without introducing noticeable differences. Based on a large set of NR simulations, the proposed fit of [57] was extended to include high-mass ratio systems, e.g. [58, 59, and reads

$$
\hat{\omega}=\hat{\omega}_{0} \sqrt{\frac{X_{B}}{X_{A}}} \frac{1+n_{1} \kappa_{2}^{T}+n_{2}\left(\kappa_{2}^{T}\right)^{2}}{1+d_{1} \kappa_{2}^{T}+d_{2}\left(\kappa_{2}^{T}\right)^{2}},
$$

with $n_{1}=3.354 \times 10^{-2}, n_{2}=4.315 \times 10^{-5}, d_{1}=7.542 \times$ $10^{-2}, d_{2}=2.236 \times 10^{-4}$. The parameter $\hat{\omega}_{0}=0.3586$ in Eq. (11) is chosen such that for equal-mass cases $q=$ $M^{A} / M^{B}=1$ and $\kappa_{2}^{T} \rightarrow 0$ the nonspinning BBH limit is recovered [57, 60.

We use Eq. 111 to determine the end of the waveform and taper the signal using a Planck taper 61. The taper begins at the estimated merger frequency and ends at 1.2 times the merger frequency. Because of the smooth frequency evolution even after the moment of merger [26, we do not expect to introduce non-negligible errors due to evaluating Eq. (6) after the merger frequency.

\footnotetext{
3 Note that this is caused by historical reasons. The NLO quadrupole-monopole term could have been added also to the other NRTidal waveform models
}

\section{TARGET HYBRID WAVEFORMS}

As mentioned in the introduction, to validate the various phasing models using the prescription given by Eq. (9) we compare them against complete BNS waveforms that are constructed stitching together the analytical waveforms constructed within the EOB approach with waveforms obtained through NR simulations. In the following three paragraphs we briefly discuss: (i) the properties of the analytical TEOBResumS EOB model; (ii) the properties of the NR waveforms; (iii) the procedure to hybridize EOB to NR waveforms in the overlapping frequency region ( $\sim$ the last 10 orbits before merger) so as to obtain complete waveforms that cover the full frequency range, from the early, quasi-adiabatic inspiral, up to the postmerger phase.

\section{A. TEOBResumS}

The TEOBResumS model is an EOB waveform model that is able to generate $\mathrm{BBH}$ waveforms through merger and ringdown, and tidally modified waveforms up to merger. The model can deal with spin-aligned binaries and is based on several recent theoretical developments [47, 48, 63, 66.

In its more recent version, the model is able to blend together, in resummed form, tidal and spin effects 37 . Notably, through a suitable modification of the concept of centrifugal radius introduced in Ref. [47, it is easily possible to incorporate the EOS dependent selfspin effects (or quadrupole-monopole terms [33]) within the EOB Hamiltonian and flux. The current version of TEOBResumS we are dealing here does this at LO only, while the EOB extension to NLO order, from the NLO spin-spin PN results of Ref. 44, 67, will be done elsewhere 68. (we recall in this respect that in the $\mathrm{BBH}$ sector NLO spin-spin interaction is already incorporated within TEOBResumS).

By contrast, we stress that some of the PN waveform approximants that we discuss below, notably TaylorF2 Tides and PhenomPv2_NRTidal, do incorporate the NLO information. We outline the importance of this difference specifically in Sec. VIB2, Note, however, that the resummation itself makes the behavior of the self-spin coupling different from the standard PN treatment, notably making it more attractive during the inspiral [37.

In addition, the spin-tidal sector of TEOBResums differs from the BBH model recently upgraded in Ref. 65] in that the effective, next-to-next-to-next-to-leading order spin-orbit parameter $c_{3}$ that is informed by NR simulation is here neglected (i.e. $\left.c_{3}=0\right){ }^{4}$

\footnotetext{
${ }^{4}$ We anyway tested that the effect of using the value of $c_{3}$ informed by $\mathrm{BBH}$ simulations [65] is essentially negligible for the range of spins considered here. This is meaningful as tidal interaction screens the effects of the high-PN correction yielded by $c_{3}$.
} 
TABLE I. BNS configurations. The name of the configuration, following the notation $\operatorname{EOS}_{M_{A} \mid M_{B}}^{\chi_{A} \mid \chi_{B}}$ is given in the first column. The subsequent columns describe the properties of the configuration: the EOS, cf. [62], the NS' individual masses $M_{A, B}$, the stars' dimensionless spins $\chi_{A, B}$, the stars' compactnesses $C_{A, B}$, the tidal deformabilities of the stars $\Lambda_{A, B}$, the tidal deformability of the binary $\tilde{\Lambda}$, the effective dimensionless coupling constant $\kappa_{\text {eff }}^{T}$, and the merger frequency $f_{\mathrm{mrg}}$ in $\mathrm{kHz}$. The last three columns give information about the NR dataset: the initial frequency of the simulation $\hat{\omega}_{0}$, the residual eccentricity [58], and the grid resolutions $\Delta x$ covering the NS.

\begin{tabular}{|c|c|c|c|c|c|c|c|c|c|c|c|c|c|c|c|}
\hline Name & EOS & $M_{A}$ & $M_{B}$ & $\chi_{A}$ & $\chi_{B}$ & $C_{A}$ & $C_{B}$ & $\Lambda_{A}$ & $\Lambda_{B}$ & $\tilde{\Lambda}$ & $\kappa_{\text {eff }}^{T}$ & $f_{\mathrm{mrg}}[k H z]$ & $\hat{\omega}_{0}$ & $e\left[10^{-3}\right]$ & $\Delta x$ \\
\hline \multicolumn{16}{|c|}{ equal mass, non-spinning } \\
\hline $2 \mathrm{~B}_{1.35 \mid 1.35}^{0.00 \mid 0.00}$ & $2 \mathrm{~B}$ & 1.3500 & 1.3500 & 0.000 & 0.000 & 0.205 & 0.205 & 127.5 & 127.5 & 127.5 & 23.9 & 2.567 & 0.038 & 7.1 & 0.093 \\
\hline SLy $_{1.35 \mid 1.35}^{0.00 \mid 0.00}$ & SLy & 1.3500 & 1.3500 & 0.000 & 0.000 & 0.174 & 0.174 & 392.1 & 392.1 & 392.1 & 73.5 & 2.010 & 0.038 & 0.4 & 0.059 \\
\hline $\mathrm{H} 4_{1.37 \mid 1.37}^{0.00 \mid 0.00}$ & $\mathrm{H} 4$ & 1.3717 & 1.3717 & 0.000 & 0.000 & 0.149 & 0.149 & 1013.4 & 1013.4 & 1013.4 & 190.0 & 1.535 & 0.037 & 0.9 & 0.083 \\
\hline \multicolumn{16}{|c|}{ equal mass, spinning } \\
\hline SLy & SLy & 1.3502 & 1.3502 & +0.052 & +0.052 & & 0.174 & 392.0 & 392.0 & 392.0 & 73.5 & 2.025 & 0.038 & 0.4 & 0.078 \\
\hline $\mathrm{SLy}_{1.35 \mid 1.35}^{0.11 \mid 0.11}$ & SLy & 1.3506 & 1.3506 & +0.106 & +0.106 & 0.174 & 0.174 & 391.0 & 391.0 & 391.0 & 73.5 & 2.048 & 0.038 & 0.7 & 0.078 \\
\hline $\mathrm{H} 4_{1.37 \mid 1.37}^{0.14 \mid 0.14}$ & $\mathrm{H} 4$ & 1.3726 & 1.3726 & +0.141 & +0.141 & 0.149 & 0.149 & 1009.1 & 1009.1 & 1009.1 & 189.2 & 1.605 & 0.037 & 0.4 & 0.083 \\
\hline \multicolumn{16}{|c|}{ unequal mass, non-spinning } \\
\hline 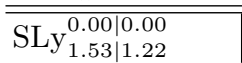 & SLy & 1.5274 & 1.2222 & 0.000 & 0.000 & 0.198 & 0.157 & 167.5 & 732.2 & 365.6 & 68.6 & 1.770 & 0.036 & 8.3 & 0.125 \\
\hline $\mathrm{SLy}_{1.65 \mid 1.10}^{0.00 \mid 0.00}$ & SLy & 1.6500 & 1.0979 & 0.000 & 0.000 & 0.215 & 0.142 & 93.6 & 1372.3 & 408.1 & 76.5 & 1.592 & 0.036 & 8.0 & 0.116 \\
\hline $\mathrm{SLy}_{1.50 \mid 1.00}^{0.00 \mid 0.00}$ & SLy & 1.5000 & 1.0000 & 0.000 & 0.000 & 0.194 & 0.129 & 192.3 & 2315.0 & 720.0 & 135.0 & 1.504 & 0.031 & 11.9 & 0.125 \\
\hline $\mathrm{MS}_{1} \mathrm{~b}_{1.53 \mid 1.22}^{0.00 \mid 0.00}$ & MS1b & 1.5278 & 1.2222 & 0.000 & 0.000 & 0.159 & 0.130 & 779.6 & 2583.2 & 1420.4 & 266.3 & 1.301 & 0.035 & 8.3 & 0.125 \\
\hline $\mathrm{MS}_{1} \mathrm{~b}_{1.65 \mid 1.10}^{0.00 \mid 0.00}$ & MS1b & 1.6500 & 1.1000 & 0.000 & 0.000 & 0.171 & 0.118 & 505.2 & 4405.9 & 1490.1 & 279.4 & 1.170 & 0.035 & 8.0 & 0.116 \\
\hline $\mathrm{MS}_{1} \mathrm{~b}_{1.500 \mid 1.00}^{0.00 \mid 0.00}$ & MS1b & 1.5000 & 1.0000 & 0.000 & 0.000 & 0.157 & 0.109 & 866.5 & 7041.6 & 2433.5 & 456.3 & 1.113 & 0.030 & 11.9 & 0.125 \\
\hline
\end{tabular}

The TEOBResums model has been validated through phase comparisons with NR simulations [10, 16]: EOB and NR waveforms are found to agree well in most regions of the BNS parameter space; slightly larger dephasing are found for models with large values of the tidal parameter (e.g., based on the MS1b EOS), suggesting that some improvements in the model are still needed. We finally recall that all EOB waveforms generated here were obtained using post-post-circular initial data consistently generalized to the spinning-tidal case [37, 69. Recently the TEOBResums model got also compared directly to the SEOBNRv4T model, see Fig. 19 of [37, and it was found a very good agreement within the parameter space region covered within this article.

\section{B. Numerical relativity waveforms}

The NR simulations used for validation of the NRTidal waveform models have been computed with the BAM code, with details given in 70 73. For all simulations, we employ the Z4c scheme [74, 75] for the spacetime evo- lution and the $1+\log$ and gamma-driver conditions $\quad 76$ 80] for the gauge system. Finite difference stencils are used for the spatial discretization of the spacetime and high resolution shock-capturing methods for the hydrodynamics part are applied. We summarize the configurations employed in this work in Tab. [1. Overall, we use 18 different physical configurations. The setups span 4 different EOSs; in particular, 2B and MS1b were chosen as relatively extreme cases to test the performance across the EOS parameter space, because both $2 \mathrm{~B}$ and MS1b are almost ruled out after the multi-messenger observation of GW170817 6]. We also test mass ratios up to $q=1.5$. While such mass ratios are possible based on binary evolution models [58, 81, no observed BNS system has such a large mass ratio 82 . Similarly, to date no NS in a BNS system has a dimensionless spin larger than $\sim 0.05$, but nevertheless we consider values up to $\chi=0.15$. All configurations employ at least three different resolutions and show phase errors at the moment of merger ranging from 0.5 to $2.5 \mathrm{rad}$ depending on the exact setup. We refer the reader to Refs. [10, 16, 26, 42] where a detailed discussion about the numerical uncertainties 
is given.

Let us emphasize that while the considered configurations cover most of the BNS parameter space which we expect to detect, for parameter estimation from $\mathrm{GW}$ observations waveforms in even larger regions need to be evaluated 5 . Consequently, one important goal for NR simulations of BNSs is to access unexplored regions in terms of masses, mass-ratios, and spins.

\section{Hybrid construction}

The procedure for hybridizing the EOB and NR waveforms is as follows. We align the EOB and NR waveforms, which employ the same binary parameters, by minimizing

$$
\mathcal{I}(\delta t, \delta \phi)=\int_{t_{i}}^{t_{f}} d t\left|\phi_{N R}(t)-\phi_{\mathrm{EOB}}(t+\delta t)+\delta \phi\right|^{2}
$$

over the frequency interval $I_{\hat{\omega}}=\left[\hat{\omega}_{i}, \hat{\omega}_{f}\right]=[0.04,0.06]$. Once the waveforms are aligned, we perform a smooth transition from the EOB data to the NR data within $I_{\hat{\omega}}$ :

$$
h_{\mathrm{hyb}}(t)=\left\{\begin{array}{lr}
h_{\mathrm{EOB}} & : \hat{\omega} \leq \hat{\omega}_{i} \\
h_{\mathrm{NR}} H(t)+h_{\mathrm{EOB}}[1-H(t)]: \hat{\omega}_{i} \leq \hat{\omega} \leq \hat{\omega}_{f} \\
h_{\mathrm{NR}} & : \hat{\omega} \geq \hat{\omega}_{f}
\end{array}\right.
$$

with the Hann window function

$$
H(t):=\frac{1}{2}\left[1-\cos \left(\pi \frac{t-t_{i}}{t_{f}-t_{i}}\right)\right],
$$

with $t_{i}, t_{f}$ denoting the times corresponding to $\hat{\omega}_{i}, \hat{\omega}_{f}$, cf. 25]. In Fig. 2 we present, as an example, the hybrid construction for the $\mathrm{SLy}_{1.35 \mid 1.35}^{0.11 \mid 0.11}$ configuration, with the alignment interval marked by vertical dashed lines.

Although the hybridization is necessary to ensure that we can use target waveforms throughout the full frequency range detectable by advanced LIGO and Virgo, the method adds further uncertainties which we shortly want to outline: While the performance of TEOBResumS has been tested [10, 16] and very good agreement has been found in the frequency interval in which the hybridization happens, we have no error measurement of

\footnotetext{
${ }^{5}$ Although potentially irrelevant for real physical systems, let us point out that for some parameter combinations, e.g., large deformabilities $\Lambda \sim 5000$ or almost extremal-antialigned spin $\chi_{A}=\chi_{B}=-0.99$ unphysical features in the waveforms can be seen. Therefore, we emphasize that interpretation of results of parameter estimation pipelines in extreme (unphysical) corners of the BNS parameters space need to be taken with care and requires special attention. However, we have not find noticeable problems for systems with spins close to the NS breakup spin $\chi \sim 0.7$, which we propose to use for an upper bound of parameter estimation runs bearing in mind that the model only got checked up to spins of $\chi=0.15$ due to missing NR simulations for high-spinning configurations.
}

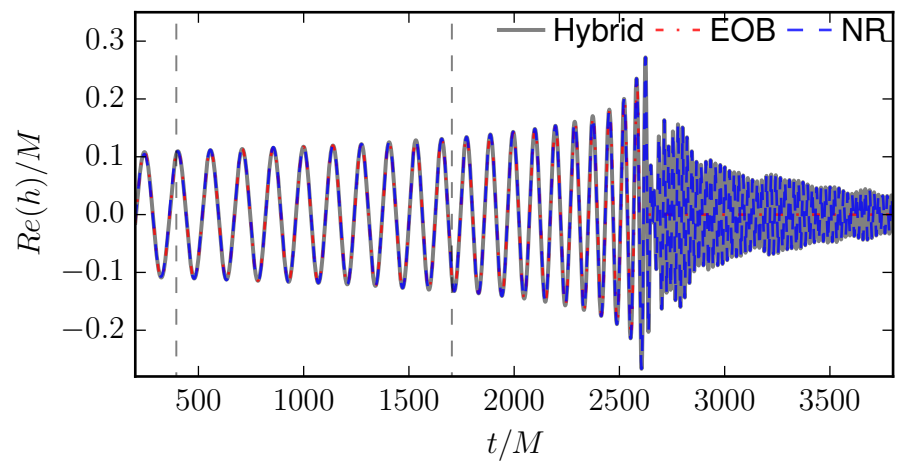

FIG. 2. Hybridization of the $\mathrm{SLy}_{1.35 \mid 1.35}^{0.11 \mid 0.11}$ configuration with the TEOBResumS model. The alignment interval is marked by vertical dashed lines. (Note that the beginning of this interval agrees with the transition between the used EOB and NR data in Fig. 11. The TEOBResumS EOB waveform is shown as a red dot-dashed curve and the NR waveform as a blue dashed curve. The final hybrid combines the long inspiral from the EOB waveform, which includes several hundred cycles (not shown in the figure), and the late inspiral, as well as the postmerger phase of the NR waveform.

the underlying analytical model at smaller frequencies, since no NR simulations are available for this frequency interval. Consequently, while NR data can be assigned with a well quantified error, see e.g. [73, the overall target waveform is missing a clear error budget. We further refer to [83] for additional details.

\section{VALIDATION OF FREQUENCY DOMAIN MODEL}

\section{A. Mismatch computation}

To quantify the performance of the NRTidal approximants, we compute the mismatch

$$
\bar{F}=1-\max _{\phi_{c}, t_{c}} \frac{\left(h_{1}\left(\phi_{c}, t_{c}\right) \mid h_{2}\right)}{\sqrt{\left(h_{1} \mid h_{1}\right)\left(h_{2} \mid h_{2}\right)}},
$$

where $\phi_{c}, t_{c}$ are an arbitrary phase and time shift, between the approximants themselves and the hybrid waveforms constructed in Sec. III. The noise-weighted overlap is defined as

$$
\left(h_{1} \mid h_{2}\right)=4 \Re \int_{f_{\min }}^{f_{\max }} \frac{\tilde{h}_{1}(f) \tilde{h}_{2}(f)}{S_{n}(f)} \mathrm{d} f .
$$

$S_{n}(f)$ gives the spectral density of the detector noise. We used the Advanced LIGO zero-detuning, high-power (ZERO_DET_high_P) noise curve of 91 for our analysis. In general, the value of $\bar{F}$ indicates the loss in signal-tonoise ratio (squared) when the waveforms are aligned in time and phase. Template banks are usually constructed such that the maximum value of $\bar{F}$ across the bank is 0.03. Although it is impossible to relate a mismatch directly to the bias obtained in parameter estimation, it is 


\begin{tabular}{|c|c|c|c|c|c|c|}
\hline \multirow[t]{2}{*}{ Model name } & \multicolumn{3}{|c|}{ BBH baseline } & \multirow[t]{2}{*}{ Tidal effects } & \multirow[t]{2}{*}{$\mathrm{QM}$} & \multirow[t]{2}{*}{ Precession } \\
\hline & $\mathrm{PP}$ & $\mathrm{SO}$ & SS & & & \\
\hline TaylorF2 & $3.5 \mathrm{PN} 84$ & $3.5 \mathrm{PN}[85,86]$ & $3 \mathrm{PN} 67,87,89$ & \multirow{2}{*}{$\begin{array}{c}x \\
7.5 \mathrm{PN}[6,49 \\
\end{array}$} & \multirow{2}{*}{$\begin{array}{c}\boldsymbol{x} \\
3 \mathrm{PN}[33,56\end{array}$} & \multirow{2}{*}{$\begin{array}{l}x \\
x\end{array}$} \\
\hline TaylorF2 Tides & $3.5 \mathrm{PN} 84$ & $3.5 \mathrm{PN}[85,86$ & $3 \mathrm{PN}$ 67, 87, 89 & & & \\
\hline TaylorT4 & $3.5 \mathrm{PN} 84$ & $3.5 \mathrm{PN}, 85,86$ & $3 \mathrm{PN}$ 67, 87, 89 & \multirow{2}{*}{$\begin{array}{c}x \\
6 \mathrm{PN}[46,49\end{array}$} & \multirow{2}{*}{\begin{tabular}{c}
\multicolumn{1}{c|}{$\boldsymbol{x}$} \\
$3 \mathrm{PN}[33,56$
\end{tabular}} & \multirow{2}{*}{$\begin{array}{l}x \\
x\end{array}$} \\
\hline TaylorT4 $4_{\text {Tides }}$ & $3.5 \mathrm{PN} 84$ & $3.5 \mathrm{PN}[85,86]$ & $3 \mathrm{PN}$ 67, 87,89] & & & \\
\hline SEOBNRv4_ROM & \multirow{2}{*}{\multicolumn{3}{|c|}{$\begin{array}{l}\text { EOB Hamiltonian with NR calibration [28, 90] } \\
\text { EOB Hamiltonian with NR calibration [28, 90] }\end{array}$}} & \multirow{2}{*}{$\begin{array}{c}\boldsymbol{x} \\
\text { NRTidal }[26] \\
\end{array}$} & \multirow{2}{*}{$\begin{array}{l}x \\
x\end{array}$} & \multirow{2}{*}{$\begin{array}{l}x \\
x\end{array}$} \\
\hline SEOBNRv4_ROM_NRTidal & & & & & & \\
\hline PhenomD & \multirow{2}{*}{\multicolumn{3}{|c|}{\begin{tabular}{|l|l|} 
TaylorF2/EOB with calibration to NR [29, 30] \\
TaylorF2/EOB with calibration to NR [29, 30]
\end{tabular}}} & $x$ & $x$ & $x$ \\
\hline PhenomD_NRTidal & & & & NRTidal 26 & $x$ & $x$ \\
\hline PhenomPv2 & \multirow{2}{*}{\multicolumn{3}{|c|}{$\begin{array}{l}\text { PhenomD } 31 \\
\text { PhenomD } 31\end{array}$}} & $x$ & $3 \mathrm{PN}, 33,56$ & $\checkmark 54,55$ \\
\hline PhenomPv2_NRTidal & & & & NRTidal 26] & $3 \mathrm{PN}[33,56$ & $\checkmark[54,55$ \\
\hline
\end{tabular}

TABLE II. Overview about the employed waveform models within this article.

in general a good measure of the performance of a particular waveform approximant, where our main interest is to understand the performance among the waveform models, i.e., how good perform the new NRTidal models with respect to the existing PN models.

\section{Variable $f_{\max }$}

In Fig. 3 we report the mismatch between the proposed model approximants, see Tab. II] and the hybrid waveforms constructed in Sec. [II] In addition to the NRTidal models and their underlying point-mass baselines, we also explore the performance of PN based models, in particular the TaylorT4 and TaylorF2 approximants (see e.g. 84, 92 94). To set the stage, we recall that we use 3.5PN-accurate expressions for the nonspinning part of the phase as well as for the spin-orbit terms 85. Up to 3PN-accurate, EOS-dependent, selfspin terms [87, 88, 95, that are essential for a conceptually meaningful comparison with the TEOBResumShybrid waveforms, are included in both TaylorF2 Tides and TaylorT4 $4_{\text {Tides }}$ approximants. For what concerns the tidal sector, while TaylorT4 Tides only incorporates the LO and NLO tidal corrections (i.e. corresponding to a $5 \mathrm{PN}$ and $6 \mathrm{PN}$ terms), in TaylorF2 we also included the $6.5,7$ and $7.5 \mathrm{PN}$ tidal terms as deduced by Taylorexpanding the tidal EOB model in Ref. [49].

Results for all approximants incorporating tidal effects are shown with solid lines: TaylorF2 2 Tides (green), TaylorT4 Tides (orange), SEOBNRv4_ROM_NRTidal (red), PhenomD_NRTidal (blue), PhenomPv2_NRTidal (cyan). Results for the corresponding approximants without tidal effects are shown with dashed lines. The mismatches in Fig. 3 are computed from $f_{\min }=30 \mathrm{~Hz}$ up to a variable maximum frequency $f_{\max }$. We mark the merger frequency extracted from the NR simulations with a vertical, black dashed line. Let us discuss the different datasets separately.

Non-spinning, equal-mass configurations: While for small tidal deformability waveform models not including tidal effects also achieve mismatches smaller $5 \times 10^{-3}$, e.g. $2 \mathrm{~B}_{1.35 \mid 1.35}^{0.00 \mid 0.00}$, this is not true for increasing tidal deformability (left to right). For stiff EOSs waveform models not including tidal effects are inaccurate and mismatches can increase more than an order of magnitude compared to NRTidal models, e.g. MS1 $\mathrm{b}_{1.35 \mid 1.35}^{0.00 \mid}$. Furthermore, mismatches between TaylorF2 Tides and the hybrid waveforms increase with increasing tidal effects (large values of $\Lambda$ ). Validating the performance of the NRTidal models among each other, we find that PhenomD_NRTidal and PhenomPv2_NRTidal tend to approximate the hybrids slightly better than the SEOBNRv4_ROM_NRTidal approximant, but differences are small.

Spinning configurations: For spinning configurations, one finds that the value of the mismatches delivered by non-tidal models is generally unacceptably large $(\gtrsim$ $1 \%$ ), and it is found to increase with the spin value (see e.g. the $\mathrm{MS}_{1} \mathrm{~b}_{1.35 \mid 1.35}$ or $\mathrm{SLy}_{1.35 \mid 1.35}$ configurations in the third and fourth row of Fig. (3)). The inclusion of EOS-dependent effects (both tidal and self-spin ones) is able to lower the mismatches to an acceptable level. Furthermore, we find that, since the various matter-dependent effects are included in TaylorF2 $2_{\text {Tides }}$ and TaylorT4 $4_{\text {Tides }}$, one also obtains an acceptable agreement $(<1 \%$ during the inspiral) with the hybrid waveform. As expected, the smallest values $(\simeq 0.1 \%)$ are obtained when the spins are small, and the EOS is soft, e.g. SLy ${ }_{1.35 \mid 1.35}^{0.05}$. This is not surprising since, for example for TaylorT4 Tides, it is known that the the point-mass (nontidal), nonspinning baseline is just by chance, especially reliable in the equal-mass, nonspinning case (see e.g. Ref. [96, 97]), although it has the property of generically underestimating the tidal forces 98 ; Furthermore, both PhenomD_NRTidal and SEOBNRv4_ROM_NRTidal exceed the $1 \%$ limit during the inspiral except for the $\mathrm{SLy}_{1.35 \mid 1.35}$ configurations where only the small-spin configuration $\mathrm{SLy}_{1.35 \mid 1.35}^{0.05 \mid 0.05}$ is around the $0.1 \%$ level. Interestingly, once the PhenomD_NRTidal model is completed 


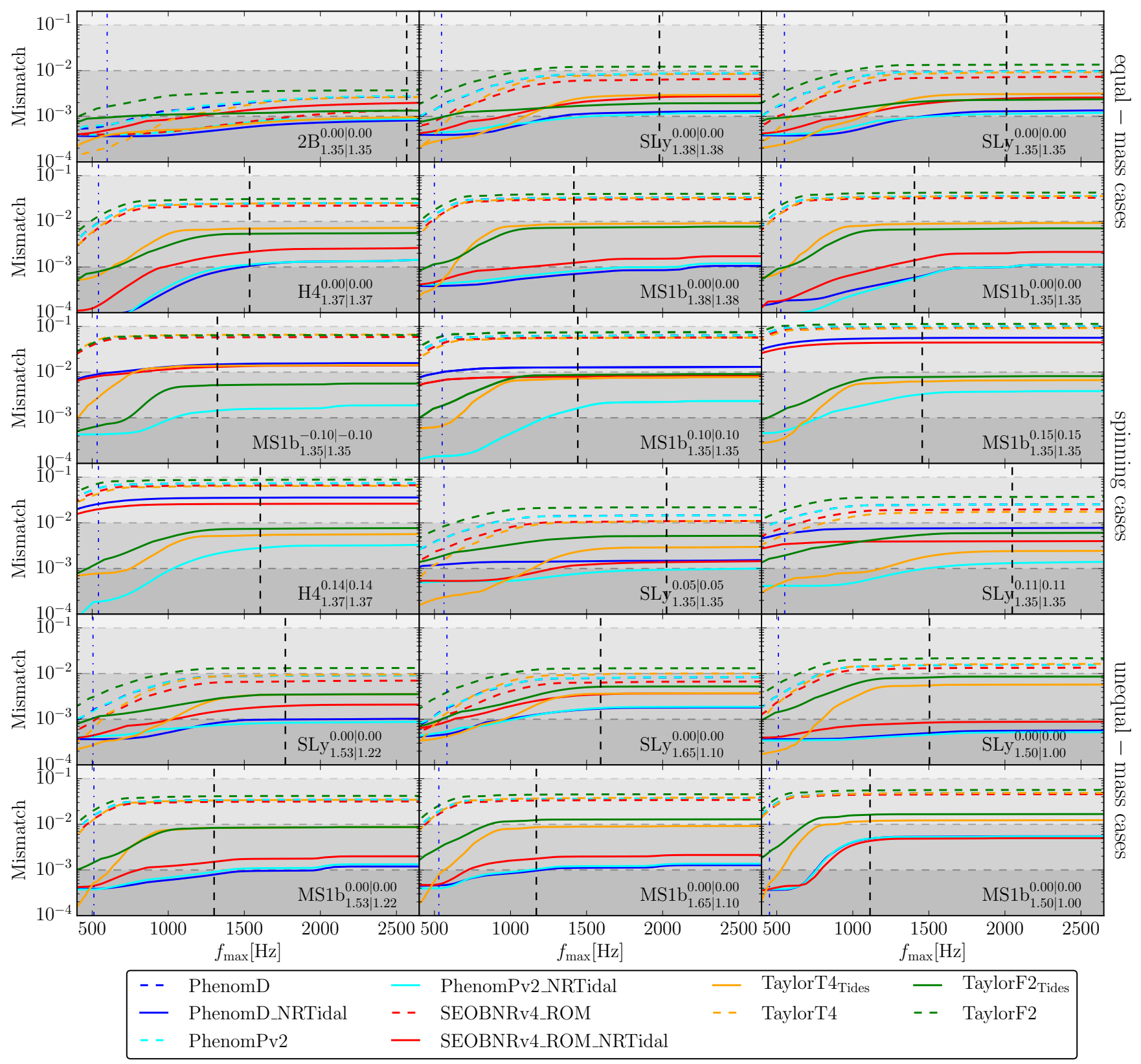

FIG. 3. Mismatches between the tidal approximants presented in this paper and the hybrid waveforms based on the configurations listed in Table I. Mismatches are computed following Eq. [15), where we set $f_{\min }=30 \mathrm{~Hz}$ and vary the maximum frequency $f_{\max }$. A black vertical dashed line marks the frequency corresponding to the moment of merger $f_{\mathrm{mrg}}$. A blue dot-dashed line marks the middle of the hybridization region between the TEOBResumS and the NR data. Note that for the analysis of GW170817 in 1], a maximum frequency of $2048 \mathrm{~Hz}$ was employed. The naming convention of the individual panels refers to the setup described in Tab. If namely: $\operatorname{EOS}_{M_{A} \mid M_{B}}^{\chi_{A} \mid \chi_{B}}$. Regarding the waveform approximants, results for all models that do not include tidal effects are marked as dashed lines, while solid lines refer to waveform models including tidal effects. The color coding is as follows: TaylorF2 $2_{\text {Tides }}$ (green), TaylorT4 ${ }_{\text {Tides }}$ (orange), SEOBNRv4_ROM_NRTidal (red), PhenomD_NRTidal (blue), PhenomPv2_NRTidal (cyan). Overall we find that the PhenomPv2_NRTidal model performs best. In particular, this model is advantageous for spinning configurations.

by the EOS-dependent self-spin terms, as it is done in the PhenomPv2_NRTidal, the mismatches drop at, or below, the $10^{-3}$ level (i.e., by up to more than an order of magnitude) for all configurations considered in the two central rows of Fig. 3 This suggests that the
PhenomPv2_NRTidal is very effective in representing the LO self-spin terms incorporated within the TEOBResumS model. Overall, our analysis shows that for sufficiently stiff EOSs, even relatively small spin magnitudes $(\sim 0.1)$ are sufficient to have an effect on mismatches between 
long signals starting at $f_{\min }=30 \mathrm{~Hz}$. By contrast, note that the mismatches are less affected by the self-spin effects for $\mathrm{SLy}_{1.35 \mid 1.35}^{0.05 \mid 0.05}$.

On the basis of this analysis, we can state that PhenomPv2_NRTidal (or similarly PhenomD_NRTidal once augmented with the EOS-dependent self-spin effects) delivers the closes matches to the EOB-NR hybrid waveforms and, thus, it is preferred with respect to the other approximants currently implemented in LAL.

Unequal mass configurations: For unequal mass, nonspinning, binaries the importance of the inclusion of tidal effects is also evident. Furthermore, we see that the TaylorF2 Tides model has the largest mismatch among all tidal approximants. Likewise the equal-mass, nonspinning case mentioned above, PhenomPv2_NRTidal and PhenomD_NRTidal are essentially equivalent.

\section{Variable $f_{\min }$}

In addition to our previous investigation, we now study the effect of varying the minimum frequency $f_{\min }$ while setting the maximum frequency $f_{\text {max }}$ equal to the merger frequency of the BNS configuration $f_{\text {mrg }}$ (dashed black lines in Fig. 3. As $f_{\text {min }}$ increases, we will be looking at a signal with decreasing length, putting more emphasis on the late-inspiral, which is generally harder to model since gravitational forces and tidal effects are stronger. Therefore, we expect that as $f_{\text {min }}$ increases, so will the mismatches. Figure 4 summarizes our results, detailed below.

Non-spinning, equal-mass configurations: In the absence of spins, we can distinguish three different regimes. (i) When tidal effects are small, cf. $2 \mathrm{~B}_{1.35 \mid 1.35}^{0.00 \mid 0.00}$, even nontidal approximants have mismatches below $10^{-2}$. Nevertheless, tidal approximants have comparatively smaller mismatches. (ii) With increasing values of $\Lambda$, non-tidal models fail to approximate the hybrid waveforms, with mismatches that easily exceed the $10^{-2}$ threshold. (iii) For even larger values of $\Lambda$, the mismatches with the TaylorF2 Tides approximant are about an order of magnitude worse than the various NRTidal models, notably even exceeding the $10^{-2}$ level.

Spinning configurations: For spinning configurations, all non-tidal models have mismatches of the order of $10^{-2}-10^{-1}$ for frequencies $f_{\min }=200 \mathrm{~Hz}$. We find that generally the PhenomPv2_NRTidal model performs best for frequencies below $f_{\min }<100 \mathrm{~Hz}$. For some setups, e.g. $\mathrm{MS}_{1.35 \mid 1.35}^{0.10 \mid 0.10}, \mathrm{SLy}_{1.35 \mid 1.35}^{0.11 \mid 0.11}$, the mismatches with respect to SEOBNRv4_ROM_NRTidal and PhenomD_NRTidal decrease as $f_{\text {min }}$ increases and have a minimum around $100-150 \mathrm{~Hz}$. We suggest that this effect is again caused by the EOS dependent spin-induced quadrupole term which is neglected in the LALSuite implementation of SEOBNRv4_ROM_NRTidal and PhenomD_NRTidal. Once $f_{\text {min }}$ is increased, the signal used for the mismatch computation becomes shorter, which in turn suppresses the error introduced by neglecting the quadrupole-monopole term. However, later in the evolution the mismatches increase again as for all other models due to inaccuracies in the description of the strong-gravity regime.

Unequal mass configurations: For unequal masses, TaylorF2 Tides produces mismatches about an order of magnitude worse than the NRTidal models. However, we also find that for high mass ratios and large tidal effects the NRTidal approximants become less accurate. In this case, e.g. MS1b $\mathrm{b}_{1.50 \mid 1.00}^{0.00 \mid 0.00}$, mismatches remain below $10^{-2}$ only if $f_{\min }$ is smaller than $100 \mathrm{~Hz}$.

\section{B. Dephasing}

In addition to mismatches, we also use phase differences computed in the frequency-domain between the hybrid waveforms and the various waveform approximants as a way to judge the performance of the waveform models. Phase differences may provide information that is complementary to the mismatch study presented in Sec. IVA for the following reasons. First, mismatches weight waveform differences according to the assumed noise spectral density and are therefore of particular importance parameter estimation. For BNS signals in Advanced LIGO, this means that mismatches are less sensitive to waveform disagreements in the late inspiral. However, we are interested in assessing the quality of the models in this regime as well.

Second, the matches we calculate are optimized over a relative time and phase shift between hybrids and models that are subject to the same amplitude and noise weighting as described above. We now apply an independent time and phase alignment in the frequency domain by minimizing the average square difference between the hybrid's phase $\left[\Psi_{1}(f)\right]$ and the model's phase $\left[\Psi_{2}(f)\right]$,

$$
\Delta \Psi_{\mathbb{L}_{2}}^{2}=\min _{t_{0}, \Psi_{0}} \int_{f_{\min }}^{f_{\max }} \frac{\left(\Psi_{1}-\Psi_{2}+2 \pi f t_{0}+\Psi_{0}\right)^{2}}{f_{\max }-f_{\min }} d f .
$$

For the optimal values of $t_{0}$ and $\Psi_{0}$ in Eq. (17), we additionally analyze the maximal value of the phase difference, $\Delta \Psi_{\max }$ [i.e., the maximum of the square root of the numerator in Eq. (17)]. Finally, we can localize the origin of the observed dephasing in an alignment-independent way by analyzing the second phase derivative (see the discussion below).

For a broadband alignment from $f_{\min }=50 \mathrm{~Hz}$ to $f_{\max }=f_{\mathrm{mrg}}$, we find that the models augmented with NR-tuned tidal phase corrections exhibit phase differences $\Delta \Psi_{\mathbb{L}_{2}} \leq 0.5$ for all hybrids except MS1b $\mathrm{b}_{1.50 \mid 1.00}^{0.00 \mid 0.00}$ for which $\Delta \Psi_{\mathbb{L}_{2}} \approx 0.7$. In order to check for localized large phase differences, we compute the maximal dephasing for all hybrids and find $\Delta \Psi_{\max }<1.3$ for all NRTidal models. These values are consistently smaller than the results for the respective point-particle baseline models. In particular, without NR-tuned tidal corrections, both $\Delta \Psi_{\mathbb{L}_{2}}$ 


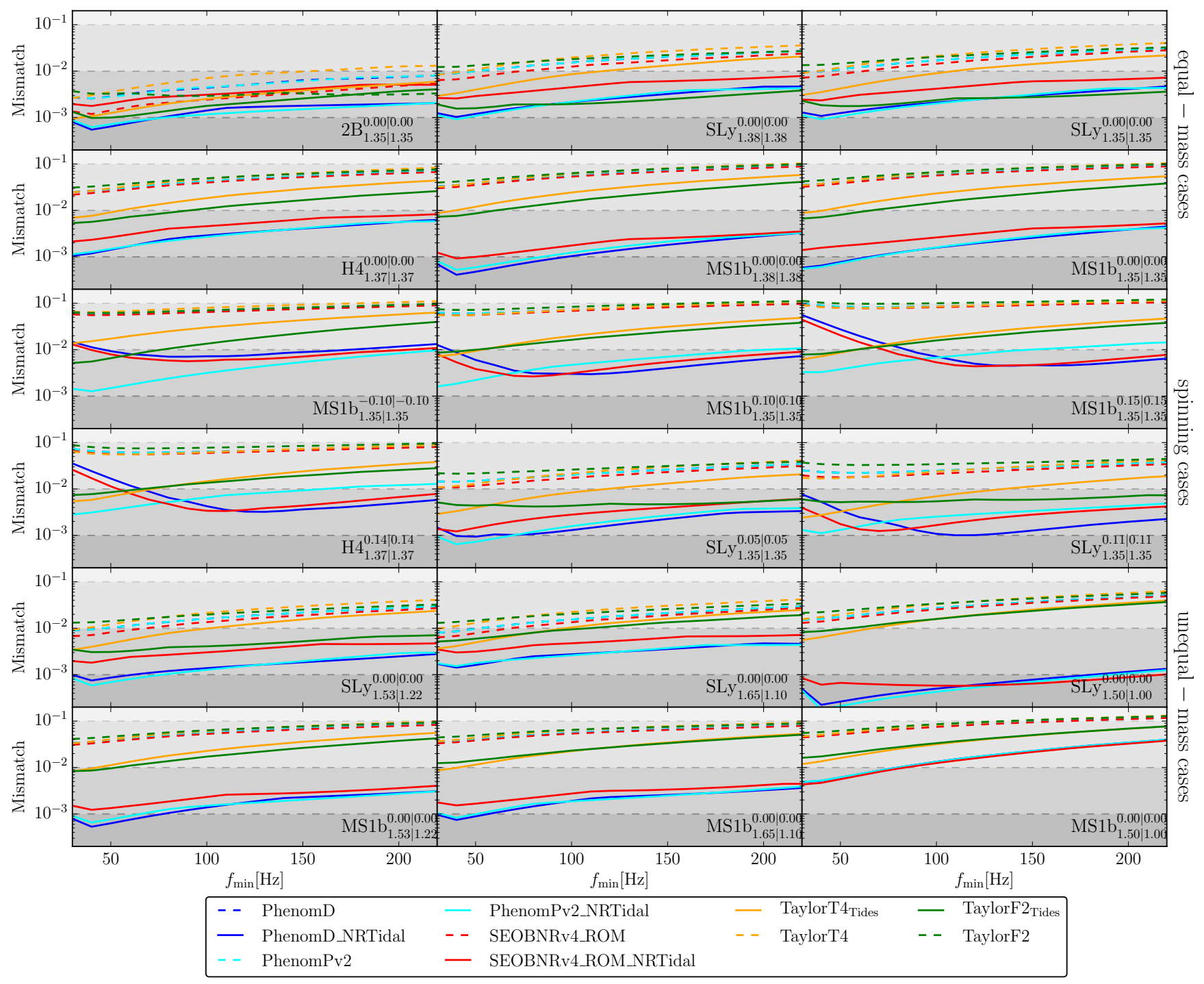

FIG. 4. Same configurations as Fig. 3. but with mismatches computed varying the minimum frequency $f_{\text {min }}$ in Eq. 15 The maximum frequency $f_{\max }$ is kept fixed and equal to the merger frequency of the BNS configurations (i.e., the values of the vertical dashed lines in the panels of Fig. 3.

and $\Delta \Psi_{\max }$ increase by a factor of $2-9$ for soft EOSs and factors of 7-23 for stiff EOSs.

Tidal PN approximants show a smaller improvement compared to their respective point-particle description, and in the case of the $2 \mathrm{~B}$ EOS, the tidal PN models even have a larger dephasing than their point-particle counterparts. In general, pure $\mathrm{PN}$ approximants perform worse or similar at best compared to the NR-tuned tidal models.

Interestingly, the broadband results discussed above do not hold universally when we align only at lower frequencies, e.g., $\left(f_{\min }, f_{\max }\right)=(50,500) \mathrm{Hz}$. In this interval, NRTidal approximants remain superior to their point-particle counterparts. However, for spinning configurations, $\mathrm{PN}$ tidal approximants now perform typically better than or as well as PhenomD_NRTidal and SEOBNRv4_ROM_NRTidal. PhenomPv2_NRTidal consis- tently shows the smallest dephasing from all hybrids. As discussed for the mismatch results, we attribute this mainly to spin-dependent quadrupole terms that are included in PhenomPv2_NRTidal and both PN approximants, but are missing in the other two NRTidal approximants in the current LALSuite implementation.

As a final corroboration of our results, we now localize the origin of the dephasing between different waveform models. Doing this based on the frequency-domain phase $\Psi(f)$ is ambiguous due to the freedom of time and phase shifting each waveform. However, the second derivate of $\Psi$ removes all degrees of freedom associated with time and phase shifts (as they manifest themselves as a linear function in the frequency domain). For signals with slowly varying amplitude, the stationary-phaseapproximation allows us to identify $d \Psi / d f$ as proportional to the time at which each frequency is realized. 


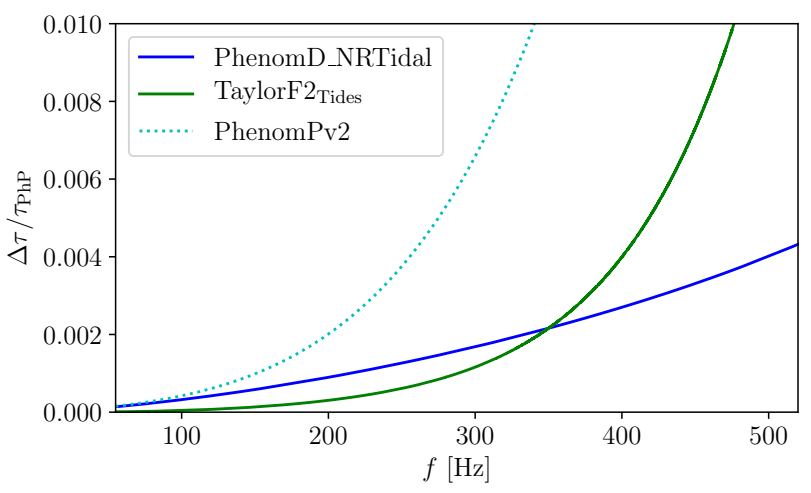

FIG. 5. Time spent by GW signals per unit frequency [see Eq. (18) and surrounding discussion], for different waveform models and shown here as relative differences to PhenomPv2_NRTidal for the MS1b $b_{1.35 \mid 1.35}^{0.15 \mid 0.15}$ case. The crossing of TaylorF2 Tides and PhenomD_NRTidal separates the frequency regimes in which either the spin-dependent quadrupole term (low frequencies) or the improved tidal corrections (high frequencies) are dominant.

Consequently,

$$
\tau(f)=\frac{d^{2} \Psi(f)}{d f^{2}}
$$

may be interpreted as the time the signal spends per unit frequency in the inspiral. (The units of $\tau$ are $\mathrm{s}^{2}$ or equivalently $\mathrm{s} / \mathrm{Hz}$.)

Figure 5 shows the relative differences in $\tau$ between frequency-domain approximants and our bestperforming model, PhenomPv2_NRTidal for MS1 $\mathrm{b}_{1.35 \mid 1.35}^{0.15 \mid 0.15}$. Not surprisingly, neglecting tidal effects completely leads to a visible disagreement across all frequencies we show (cf. PhenomPv2 curve). Interestingly, below $\sim 350 \mathrm{~Hz}$, we see that TaylorF2 Tides agrees better with PhenomPv2_NRTidal than PhenomD_NRTidal does. We stress again that this is due to missing, spin-dependent quadrupole terms in PhenomD_NRTidal. Above $\sim 350 \mathrm{~Hz}$, however, the improved NR-tuned tidal corrections are more important than the quadrupole terms, which leads to a rapid decline in accuracy in TaylorF2 $2_{\text {Tides }}$, while PhenomD_NRTidal agrees better with PhenomPv2_NRTidal at those frequencies.

\section{COMPARISON WITH TIME DOMAIN WAVEFORMS}

In the following, we also want to to test the performance of the waveform models in the time domain. While this analysis is less relevant for unbiased parameter estimation, since no information about the noise spectral density enters in the computation of the phase difference, we quantify the time domain behavior to fully assess the quality of our GW approximants. For this purpose we compute via inverse Fourier transformation the waveform strain $h(t)$. Although it is not possible to correlate phase differences directly to possible biases during the estimation of source properties, one can expect that waveform models with small phase differences will allow a more accurate interpretation of measured GW signals. Consequently, our main focus will be the performance of the employed waveform models, Tab. II among each other.

As representative cases, we show the equal-mass nonspinning $\mathrm{SLy}_{1.35 \mid 1.35}^{0.00 \mid 0.00}$, the equal-mass spinning $\mathrm{H} 4_{1.37 \mid 1.37}^{0.14 \mid 0.14}$, and the non-equal mass non-spinning $\mathrm{MS}_{1} \mathrm{~b}_{1.65 \mid 1.10}^{0.00}$, but similar results are obtained for other configurations. We focus on two different comparisons: (i) We align waveforms computed from different approximants with the hybrid waveform several hundred orbits before the actual merger. At this stage one could expect that all models allow a reasonable prescription of the binary dynamics and the alignment procedure is justified. (ii) We align the waveforms obtained from different waveform approximants with the hybrid waveforms about 15 orbits before the merger. At this time tidal effects influence the binary dynamics and the alignment procedure using non-tidal waveforms is purely artificial (see discussion below).

Both time-domain alignment procedures are different from the ones carried out in the frequency domain. While in the frequency domain phase difference and mismatch computations are usually computed over the entire frequency interval, in the time domain we aim at studying the accumulation of errors during the binary evolution.

As a final check we also compare the precessing PhenomPv2_NRTidal model with a precessing NR waveform of [99. While this comparison is limited to the last 15 orbits before merger, it provides a first qualitative assessment of the accuracy of the PhenomPv2_NRTidal model for precessing systems.

\section{A. Waveform alignment in the early inspiral}

We consider the last $58 \mathrm{~s}$ before the merger. During this time the NSs complete $\sim 1400$ orbits, where the exact number depends on the configuration details. The time-domain dephasing $\Delta \phi=\phi_{\text {hybrid }}-\phi_{\text {model }}$ is shown on a logarithmic scale in the left panels of Fig. 6 and on a linear scale focusing on the last few orbits in the right panels. We align the waveforms in the time interval $t \in[-58 \mathrm{~s},-40 \mathrm{~s}]$, where $t=0 \mathrm{~s}$ marks the end of the inspiral of the hybrid. The color coding of the waveform approximants is identical to the previous figures. We will now discuss each individual waveform separately.

Non-spinning, equal mass $\left(\mathrm{SLy}_{1.35 \mid 1.35}^{0.00}\right)$ : Over the time interval considered, the NSs perform 1388 orbits, i.e., the full signal contains $2775 \mathrm{GW}$ cycles (a total of $17434 \mathrm{rad})$.

The phase differences between all models and the hybrid waveform is below $30 \mathrm{rad}$; as shown in the right, top panel of Fig. 6, most of the phase difference is ac- 


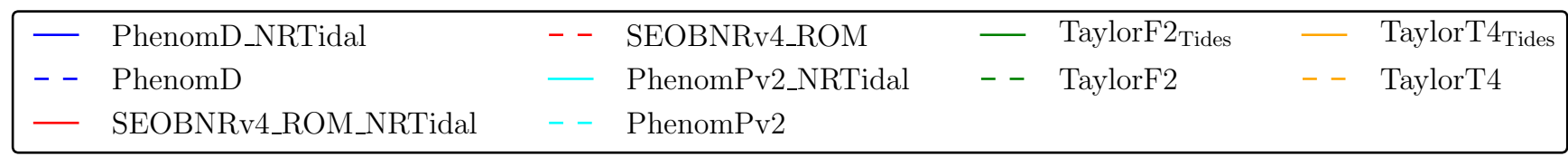
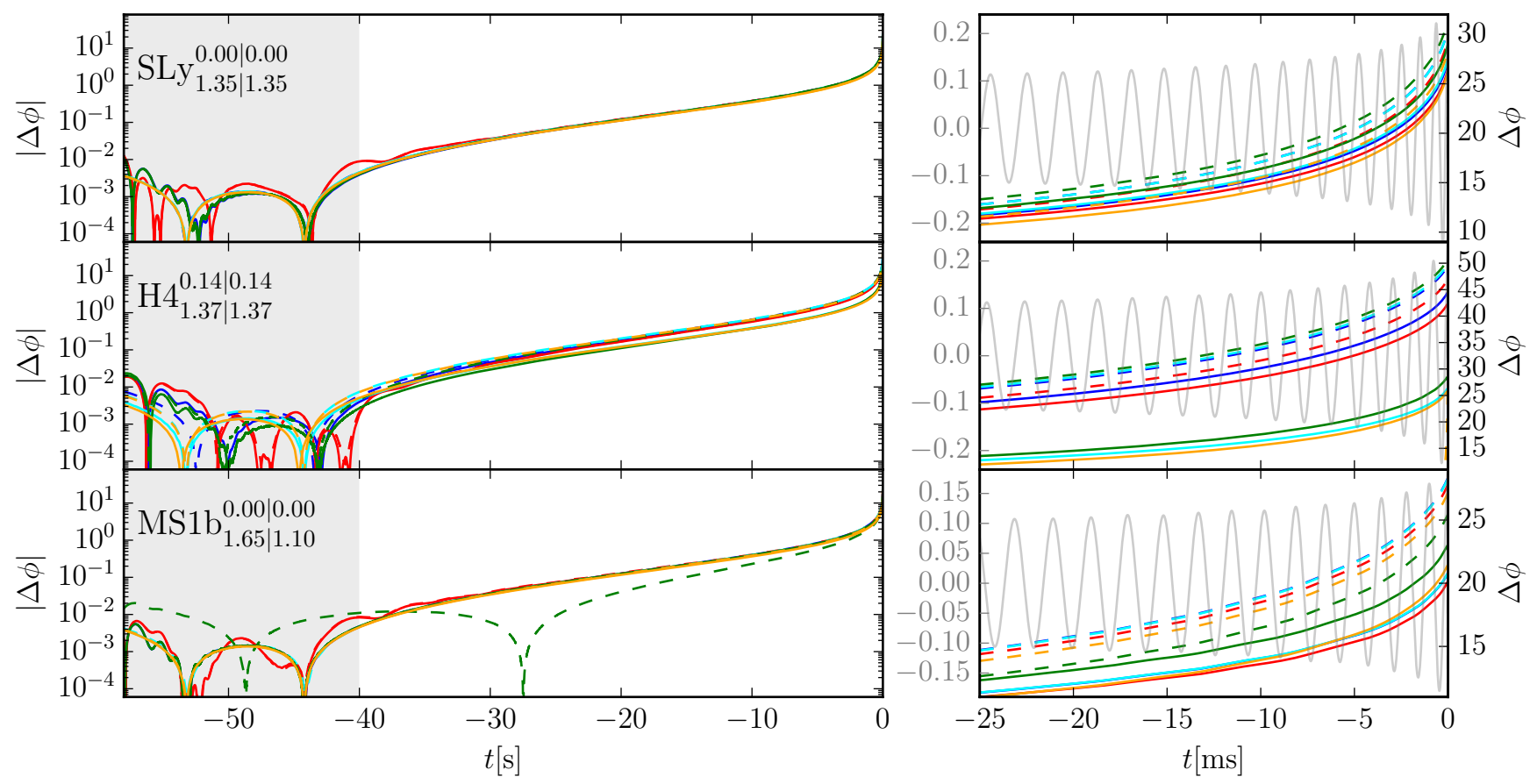

FIG. 6. Time domain dephasing $\Delta \phi$ between waveform models and hybrids for $\mathrm{SLy}_{1.35 \mid 1.35}^{0.00 \mid 0.00}$ (top panels), H4 $4_{1.37 \mid 1.37}^{0.14 \mid 0.14}$ (middle panels), $\mathrm{MS}_{1} \mathrm{~b}_{1.65 \mid 1.10}^{0.00 \mid 0.00}$ (bottom panels). Dashed lines refer to dephasings obtained from waveform models not incorporating tidal effects, while solid lines include tidal effects (the color coding is the same as in Fig. 3). The hybrid waveforms and model waveforms are aligned according to Eq. $[12$ in the time interval $t \in[-58 \mathrm{~s},-40 \mathrm{~s}]$ before the merger, cf. gray shaded region. The right panels show only the last $25 \mathrm{~ms}$ before merger, i.e., the last few gravitational wave cycles, and the real part of the hybrid waveforms is shown in gray for a better visual interpretation.

cumulated during the last $\sim 15 \mathrm{GW}$ cycles 6 , For all models considered the difference between tidal and nontidal waveforms is small: $\lesssim 1.5$ full GW cycles. While almost all waveform models incorporating tidal effects perform equally well, the TaylorF2 2 ides model has the worst performance, while SEOBNRv4_ROM_NRTidal and TaylorT4 $4_{\text {Tides }}$ perform best.

Spinning, equal mass $\left(\mathrm{H} 4_{1.37 \mid 1.37}^{0.14 \mid 0.14}\right)$ : The overall phase accumulated in the total time interval is about $17272 \mathrm{rad}$, which corresponds to 1378 orbits, or a total of $2750 \mathrm{GW}$ cycles before the merger of the two stars. Due to the larger effective tidal coupling constant $-\kappa_{\text {eff }}^{T}=189.2$, as opposed to $\kappa_{\text {eff }}^{T}=73.5$ for the $\mathrm{SLy}_{1.35 \mid 1.35}^{0.00 \mid 0.00}$ case we also find larger phase differences between PhenomD,

\footnotetext{
${ }^{6}$ We emphasize again that the exact phase difference is subject to the exact alignment procedure and window. Furthermore, since no information about the noise spectral density enters in the computation, the phase difference can not be directly related to the mismatch or the effect on full parameter estimation. However, it is a crucial quantity for a full assessment of the GW model quality.
}

SEOBNRv4_ROM and their NRTidal counterparts. However, the main phase difference caused by matter effects comes from the spin induced quadrupole moment which effects the dynamics significantly earlier than the tidal contributions modeled in PhenomD_NRTidal and SEOBNRv4_ROM_NRTidal. Therefore, phase differences of about $20 \mathrm{rad}$ between PhenomPv2 and PhenomPv2_NRTidal are obtained. Indeed the effect of the spin induced quadrupole contribution is already visible about $30 \mathrm{~s}$ before the merger, cf. left, middle panel. Consequently, for an accurate description of the entire GW signal for spinning NSs we do emphasize again the importance of incorporating the EOS dependent contributions which are coupled to the star's intrinsic rotation.

Non-spinning, unequal mass $\left(\mathrm{MS}_{1} \mathrm{~b}_{1.65 \mid 1.10}^{0.00 \mid 0.00}\right)$ : The $\mathrm{MS}_{1} \mathrm{~b}_{1.65 \mid 1.10}^{0.00 \mid 0.00}$ configuration accumulates $17642 \mathrm{rad}$ before the moment of merger, which corresponds to 1390 orbits, i.e. $2780 \mathrm{GW}$ cycles. Overall, phase differences between models that incorporate tidal effects and those that do not are of the order of $10 \mathrm{rad}$. Comparing the performance of tidal waveform models, the TaylorF2 Tides model's performance is again the worst. The best perfor- 
mance is obtained by the SEOBNRv4_ROM_NRTidal model, with a phase difference of about $20 \mathrm{rad}$ compared to the hybrid waveform. Since this configuration contains irrotational NSs, no phase difference is visible in the early inspiral between the different approximants, see bottom left panel.

\section{B. Waveform alignment in the strong-field regime}

We also analyze the performance of the waveform approximants focusing on the last orbits before merger. For this purpose we align the waveforms in the time interval $t \in[-50 \mathrm{~ms},-40 \mathrm{~ms}]$ before the merger. Most NR simulations have an inspiral shorter than $50 \mathrm{~ms}$, see, e.g., [25, 99 101] for exceptions. Consequently, the following analysis is similar to assessing the quality of the waveform approximant purely based on NR simulations. Let us further emphasize that aligning different waveforms artificially in a regime in which they disagree can lead to spurious artifacts. For this purpose, we also present the dephasing over the entire region in the left panels of Fig. 7. As done previously, we discuss the three representative examples individually.

Non-spinning, equal mass $\left(\mathrm{SLy}_{1.35 \mid 1.35}^{0.00 \mid 00}\right)$ : Once the waveforms are aligned in the late inspiral, a clear separation between waveform models that incorporate tidal effects and ones that do not takes place. All nontidal approximants have phase differences of the order of $>6$ rad at merger. The PN approximants TaylorF $2_{\text {Tides }}$ and Taylor 14 Tides also have phase differences with respect to the hybrid of the order of $\sim 5 \mathrm{rad}$; additionally, TaylorF2 2 Tides stops before the actual merger. The NRTidal models achieve phase accuracies of $\lesssim 2$ rad before the merger, with SEOBNRv4_ROM_NRTidal performing best. In light of the phase differences before the alignment window, we find that $\Delta \phi$ is negative and of the order of $\sim 1$ rad.

Spinning, equal mass $\left(\mathrm{H} 4_{1.37 \mid 1.37}^{0.14 \mid 0.14}\right)$ : We find that for this configuration it is not possible to align the non-tidal waveforms and the hybrid waveform in a sensible way: in other words, non-tidal waveforms cannot describe the BNS system at times about $\sim$ 10 orbits before the merger. Furthermore, Fig. 7 (middle row) emphasizes again the importance of the spin-induced and EOS dependent quadrupole term incorporated in the PhenomPv2_NRTidal, TaylorF2 ${ }_{\text {Tides }}$, TaylorT4 Tides models. Overall, for this configuration the PhenomPv2_NRTidal model performs best with a phase difference of about 2 rad at merger.

Non-spinning, unequal mass $\left(\mathrm{MS}_{1} \mathrm{~b}_{1.65 \mid 1.10}^{0.00 \mid 0.00}\right)$ : As for the previous case, the non-tidal waveform models do not allow a proper alignment within the time interval $t \in[-50,-40] \mathrm{ms}$. This is due to the large tidal effects for this particular configuration, which are driven by an effective tidal coupling constant of $\kappa_{\text {eff }}^{T}=279.4$. Additionally, we note that the performance of the TaylorT $4_{\text {Tides }}$ model is worse than the TaylorF $2_{\text {Tides }}$ model for unequal masses and that the TaylorF2 Tides model stops a few cycles before the actual merger. The best performances are achieved by the PhenomPv2_NRTidal, PhenomD_NRTidal and SEOBNRv4_ROM_NRTidal approximants, with a phase difference are merger below $2 \mathrm{rad}$.

\section{Precessing Waveform Comparison}

As a final check, we test the performance of PhenomPv2_NRTidal for a precessing BNS configuration. Due to the absence of a tidal EOB model including precession effects, we restrict our analysis to the last 15 orbits covered by the NR simulation of 99 . The specific configuration we investigate consists of NSs with masses $M_{A}=1.3553$ and $M_{B}=1.1072$, tidal deformabilities $\Lambda_{A}=382.3$ and $\Lambda_{B}=1308.6$, dimensionless spins $\chi_{A}=(-0.077,-0.077,-0.077)$ and $\chi_{B}=$ $(-0.089,-0.089,-0.089)$, and SLy EOS. The NR simulation starts at a GW frequency of $392 \mathrm{~Hz}$.

We present the waveform strain for an inclination of $0^{\circ}$ in the top two panels of Fig. 8, and for an inclination of $90^{\circ}$ in the bottom panels. We assume a distance to the system of $100 \mathrm{Mpc}$ as in 99. Overall we find phase differences for $\iota=0^{\circ}$ below one radian, which is of the order of the uncertainty in the NR simulation [99. To compare the waveforms, we vary the initial frequency for the PhenomPv2_NRTidal model and align the waveforms for $\iota=0^{\circ}$ at the peak amplitude followed by an additional time shift to minimize the phase difference. The same overall time shift is then also employed for the $\iota=90^{\circ}$ orientation.

Considering the amplitude difference, we do find that the PhenomPv2_NRTidal waveform has a larger amplitude close to the merger, which is caused by the missing amplitude corrections due to tidal effects in the NRTidal approach. In the bottom two panels, GW polarizations $h_{+}, h_{\times}$for an inclination of $\iota=90^{\circ}$ are presented. The plus-polarization shows a similar trend as for $\iota=0$, i.e, the amplitude of PhenomPv2_NRTidal is overestimated, while the overall phasing is in good agreement. Finally, for $\iota=90^{\circ}$ precession effects are clearly visible in $h_{\times}$. The precession cycle is recovered by the PhenomPv2_NRTidal waveform which verifies the assumption that tidal effects and precession effects decouple at LO. Interestingly, throughout the inspiral the amplitude seems to be underestimated by the PhenomPv2_NRTidal model, while the opposite happens at the moment of merger. Additionally, we also find that for all inclinations and polarizations the merger time (conventionally taken as the maximum of the amplitude) of the PhenomPv2_NRTidal model determined by Eq. (11) is consistent with the merger of the NR simulation.

Overall, bearing in mind the difficulties and uncertainties in the extraction of spin from the NR simulations and the short length of the (single) NR waveform, we conclude that the PhenomPv2_NRTidal model also seems 


\begin{tabular}{|c|c|c|c|c|c|c|c|}
\hline - & PhenomD_NRTidal & -- & SEOBNRv4_ROM & - & TaylorF2 Tides & - & TaylorT4 Tides \\
\hline-- & PhenomD & - & PhenomPv2_NRTidal & -- & TaylorF2 & -- & TaylorT4 \\
\hline - & SEOBNRv4_ROM_NRTidal & -- & PhenomPv2 & & & & \\
\hline
\end{tabular}
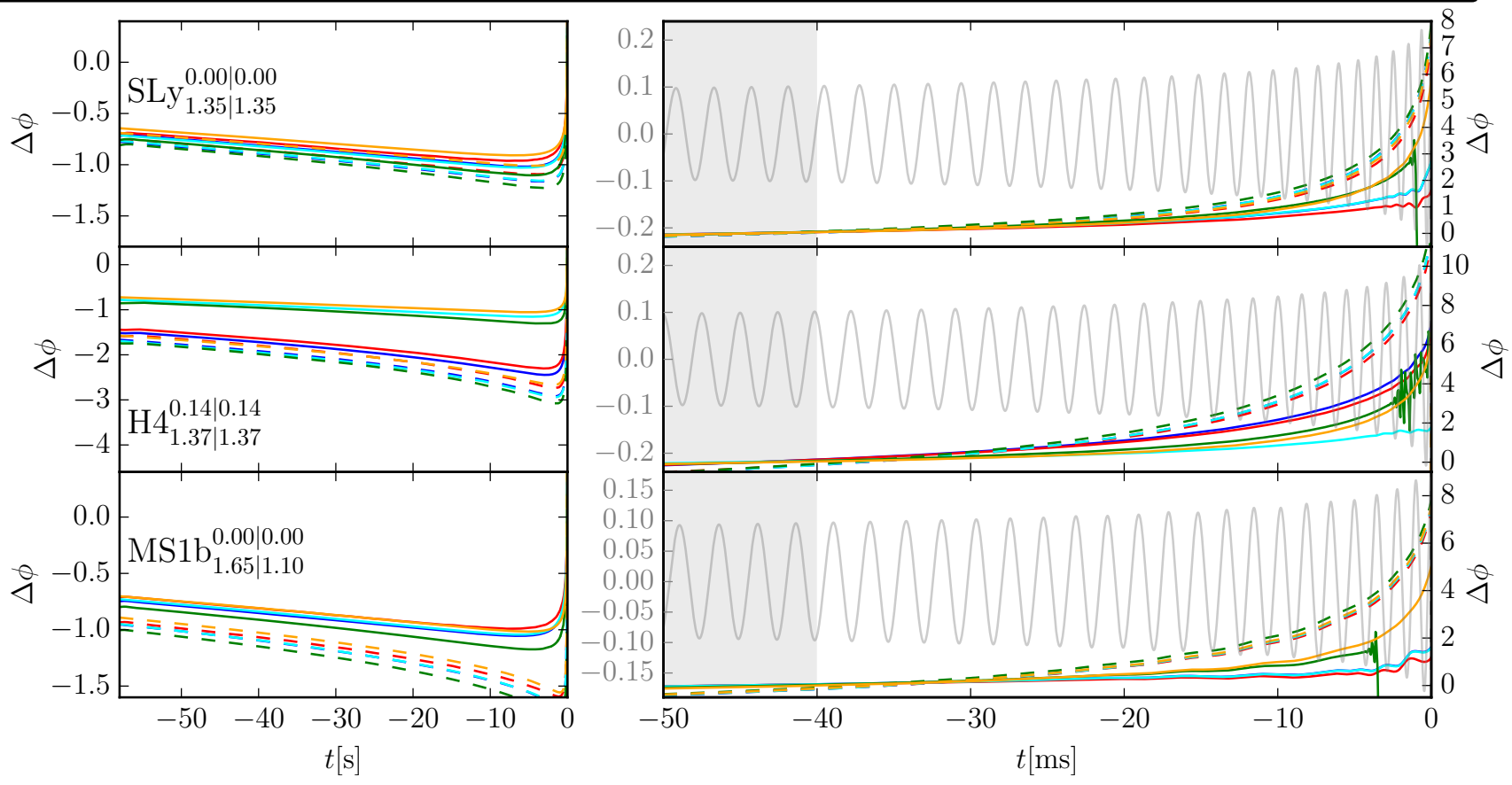

FIG. 7. Time domain dephasing $\Delta \phi$ between waveform models and hybrids for $\mathrm{SLy} \mathrm{L}_{1.35 \mid 1.35}^{0.00 \mid 0.00}$ (top panels), $\mathrm{H} 4_{1.37 \mid 1.37}^{0.14 \mid 0.14}$ (middle panels), MS1b $\mathrm{b}_{1.65 \mid 1.10}^{0.00 \mid 0.00}$ (bottom panels), similar to Fig. 6 but this time aligned in the time interval $t \in[-50 \mathrm{~ms},-40 \mathrm{~ms}]$ before merger, cf. gray shaded region.

able to deliver a consistent representation of the waveform of precessing systems up to merger.

\section{SYSTEMATICS EFFECTS IN NRTIDES AND THEIR IMPLICATIONS}

\section{A. Analytical tidal knowledge beyond} next-to-leading order and its relation with NRTides

We want to stress that while the NRTidal approximant is constructed to reproduce the known NLO tidal knowledge, analytical knowledge beyond NLO exists [49. The particular choice for the form of Eq. (5) and the restriction to NLO was made because of simplicity and to allow a smaller number of parameters in the rational function used for fitting.

However, analytical information beyond NLO incorporated for example in state-of-the-art tidal EOB models like TEOBResumS have been important to achieve good agreement between EOB and NR waveforms 16. More precisely, the analytical tidal information currently available and relevant here is: the full next-to-next-to-leading order tidal contribution to the interbody EOB interaction potential computed in 102 (i.e., formally a 7PN contribution) as well as (ii) tail terms that can be obtained, at arbitrary PN order, by expanding the resummed tail factor entering the resummed EOB waveform [49, 103] and (iii) gravitational-self-force contributions to the interaction potential obtained at high PN order and suitably resummed [104]. In particular, putting together some of the available analytic information, Ref. 49] obtained the tidal phase at global 7.5PN order that is incorporated in TaylorF2 and that is fully known analytically except for a $7 \mathrm{PN}$ waveform amplitude coefficient. In this respect it was pointed out that such, yet uncalculated, $7 \mathrm{PN}$ tidal coefficient $\beta_{2}^{22}$ entering the quadrupolar waveform is very likely negligible not only with respect to the other tidal ones (notably the dynamical ones), but also with respect to the corresponding $2 \mathrm{PN}$ point-mass coefficient. The arguments of Ref. [49] illustrate that it might be possible to improve the fitting ansätze mentioned above in Eqs. (5)-(7) by imposing not only the $6 \mathrm{PN}$ term, but also the $6.5 \mathrm{PN}$ and $7.5 \mathrm{PN}$ ones (that are analytically fully known) as well as the 7PN one that is currently lacking the waveform amplitude contribution $\beta_{2}^{22}$ mentioned above7.

\footnotetext{
7 In this respect, one has to remind that one could obtain the TaylorF2 tidal approximant expanding the EOB analytic phasing to even higher PN order.
} 


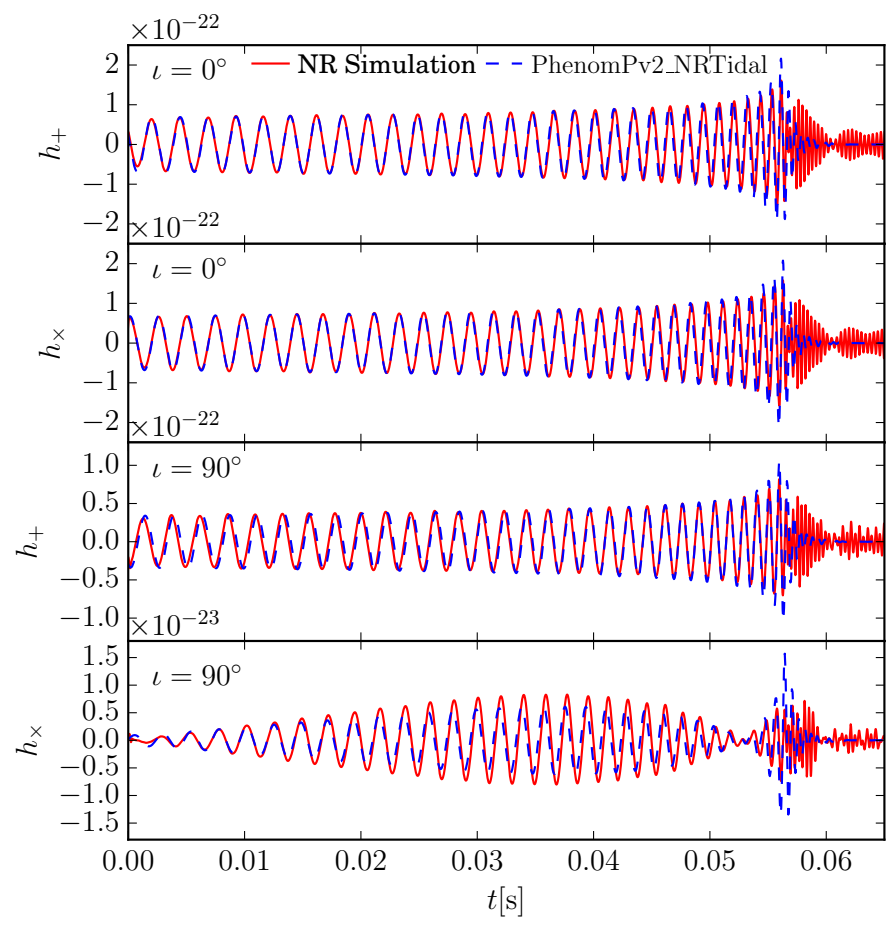

FIG. 8. GW strain for a precessing NR simulation of 99 (red, solid) for SLy EOS (see text for details) and the PhenomPv2_NRTidal model (blue, dashed). Results for zeroinclination (face on) are shown in the two top panels; results for an inclination of $90^{\circ}$ (edge on) are shown in the bottom panels. We assume a distance to the binary of $100 \mathrm{Mpc}$.

As an example that illustrates how the current fits, Eqs. (5)-(6), differ from the analytically known expression, let us expand $P_{\Psi_{2.5 \mathrm{PN}}}^{\mathrm{NRTidal}}$ in powers of $x$ up to $2.5 \mathrm{PN}$ order. One finds

$P_{\Psi_{2.5 \mathrm{PN}}}^{\mathrm{NRTidal}}=1+\frac{3115}{1248} x-4.22 x^{3 / 2}+23.32 x^{2}-111.84 x^{5 / 2}$,

while the analytically expression of Ref. 49, restricted to the equal-mass case, reads

$$
\begin{aligned}
\hat{\Psi}_{2.5 \mathrm{PN}}^{\mathrm{T}} & =1+\frac{3115}{1248} x-\pi x^{3 / 2}+\left(\frac{28024205}{3302208}+\frac{20}{351} \beta_{2}^{22}\right) x^{2} \\
& -\frac{4283}{1092} \pi x^{5 / 2} \approx 1+\frac{3115}{1248} x-\pi x^{3 / 2} \\
& +\left(8.491+0.057 \beta_{2}^{22}\right) x^{2}-12.32 x^{5 / 2}
\end{aligned}
$$

One sees here that the (relative) $1.5 \mathrm{PN}$ tidal term incorporated in NRTidal is about $30 \%$ smaller than the correct analytical one, while the $2.5 \mathrm{PN}$ one is even 9 times smaller. Assuming, as argued in Ref. 49, that the contribution due to the yet uncalculated waveform amplitude coefficient $\beta_{22}^{2}$ can be neglected, the $2 \mathrm{PN}$ term is approximately 2.7 times larger than the corresponding analytical value. This illustrate the strong "effectiveness" of the NRTidal model already in the PN-regime, with highorder (effective) PN terms that are required to fix the imperfect value of the low PN ones. The lack of the cor-

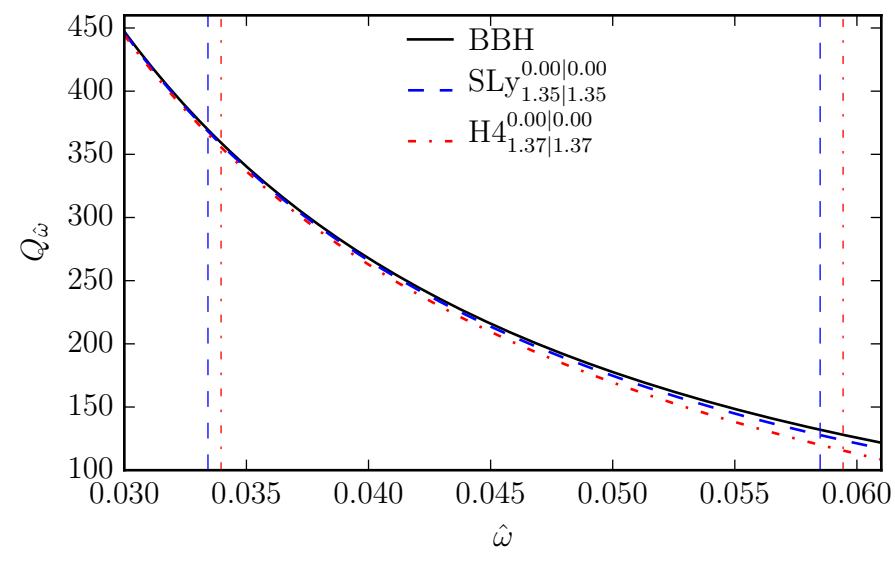

FIG. 9. Computation of the invariant characterization of the phasing of TEOBResumS, $Q_{\hat{\omega}}$, Eq. 21) for an equal-mass, nonspinning, BBH and for two of the configurations considered. The effect of tides pushes the BBH curve down. Differences that looks small on this scale actually correspond to several radians accumulated in phase difference. The vertical dashed lines refer to 400 and $700 \mathrm{~Hz}$ for the two different systems.

rect low-frequency behavior beyond NLO is per se not a big concern as the approximant should always be used as a whole; still our analysis illustrates its effective nature that should be kept in mind. Consequently, although the current NRTidal approximant yields rather small mismatches, for current standards, with TEOBResums-based hybrids, we plan to improve the NRTidal model in the near future by including beyond-NLO effects.

\section{B. $Q_{\hat{\omega}}-$ phasing analysis}

\section{Contributions due to tidal effects}

Let us discuss, from a different perspective, how the tidal phasing yielded by the NRTidal model compares with the one of the non-hybridized TEOBResumS model. This comparison is especially interesting at low frequencies, a regime that cannot be touched by NR simulations. To be conservative, we shall investigate and discuss this comparison up to dimensionless GW frequency $\hat{\omega}=0.06$, which is the upper limit of the frequency interval where the hybridization is done.

To do the comparison in a straightforward way, we build on previous work [22, 98] by using the dimensionless function $Q_{\hat{\omega}}$ 93. defined as

$$
Q_{\hat{\omega}}=\frac{\hat{\omega}^{2}}{\partial_{t} \hat{\omega}} .
$$

This function has several properties that will be useful in the present context. First, its inverse can be considered as an adiabatic parameter $\epsilon_{\text {adiab }}=1 / Q_{\hat{\omega}}=\partial_{t} \hat{\omega} / \hat{\omega}^{2}$ whose magnitude controls the validity of the stationary phase approximation (SPA) that is normally used to compute the frequency-domain phasing of $\mathrm{PN}$ approximants 
during the quasi-adiabatic inspiral. Thus, the magnitude of $Q_{\hat{\omega}}$ itself tells us to which extent the SPA delivers a reliable approximation to the exact Fourier transform of the complete inspiral waveform, that also incorporates nonadiabatic effects. Let us recall 49 that, as long as the SPA holds, the phase of the Fourier transform of the time-domain quadrupolar waveform

$$
\tilde{h}_{22}(f) \equiv \tilde{A}(f) e^{-\mathrm{i} \Psi(f)}
$$

is simply the Legendre transform of the quadrupolar time-domain phase $\phi(t)$, that is

$$
\Psi_{\mathrm{SPA}}(f)=2 \pi f t_{f}-\phi\left(t_{f}\right)-\frac{\pi}{4},
$$

where $t_{f}$ is the solution of the equation $\hat{\omega}\left(t_{f}\right)=2 \pi f$. Differentiating the above equation one then finds

$$
\frac{d^{2} \Psi_{\mathrm{SPA}}}{d \hat{\omega}_{f}^{2}} \hat{\omega}_{f}^{2}=Q_{\hat{\omega}}\left(\hat{\omega}_{f}\right)
$$

where now $\hat{\omega}_{f}=2 \pi f$ is the Fourier domain circular frequency that coincides, because of the SPA, with the timedomain frequency $\hat{\omega}(t)$. Second, the integral of $Q_{\hat{\omega}}$ per logarithmic frequency yields the phasing accumulated by the evolution on a given frequency interval $\left(\hat{\omega}_{L}, \hat{\omega}_{R}\right)$, that is

$$
\Delta \phi\left(\hat{\omega}_{L}, \hat{\omega}_{R}\right) \equiv \int_{\hat{\omega}_{L}}^{\hat{\omega}_{R}} Q_{\hat{\omega}} d \log \hat{\omega} .
$$

Additionally, since this function is free of the two "shift ambiguities" that affect the GW phase (either in the time or frequency domain), it is perfectly suited to compare in a simple way different waveform models $[10,22,69,98]$, We start by computing $Q_{\hat{\omega}}$ directly from the timedomain phasing of TEOBResumS. We consider two, equalmass, configurations $\mathrm{SLy}_{1.35 \mid 1.35}^{0.00 \mid 0.00}$ and $\mathrm{H} 4_{1.37 \mid 1.37}^{0.00 \mid 0.00}$ starting at $30 \mathrm{~Hz}$ as well as the corresponding $\mathrm{BBH}$ one. Though the calculation of $Q_{\hat{\omega}}$ is, per se, straightforward, since one only has to compute time-derivatives of $\phi(t)$, in practice there are subtleties that one has to take into account. First of all, any residual eccentricity related to slightly inconsistent set up of the initial data of TEOBResumS will show up as oscillations in the curve (with typically larger amplitudes the lower the initial frequency is) preventing one from using this diagnostics for quantitative comparisons. To avoid this, TEOBResumS implements post-post-circular initial data [69] that are able to deliver eccentricity-free evolutions. In addition, the time-domain oversampling of the inspiral may result in high-frequency numerical noise from the computation of numerical derivatives, that typically hides the lowfrequency behavior of the curve, preventing, for instance,

\footnotetext{
8 As a quantitative reference for the reader, we note that a difference in $Q_{\hat{\omega}}$ of order unity sustained on a frequency interval that doubles amounts in 1 rad dephasing.
}

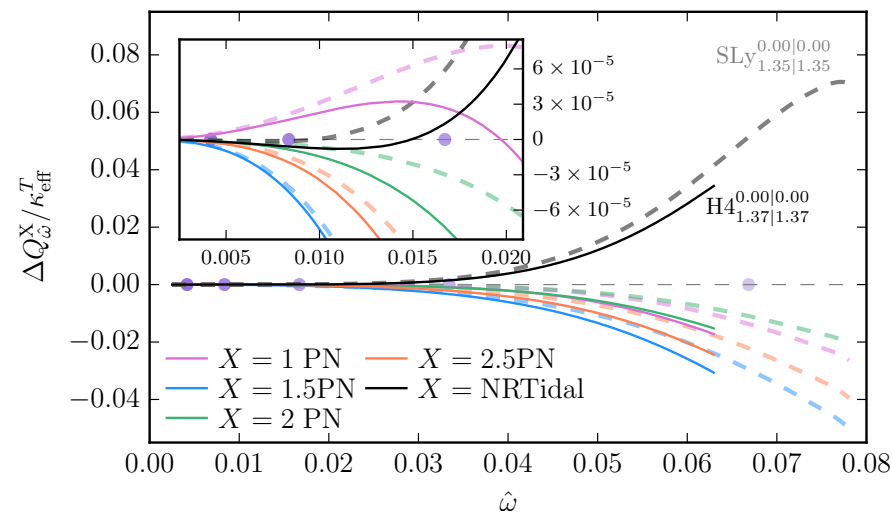

FIG. 10. $Q_{\hat{\omega}}$-description of the tidal phasing for $\mathrm{SLy}_{1.35 \mid 1.35}^{0.00 \mid 0.00}$ (dashed lines) and $\mathrm{H} 4_{1.37 \mid 1.37}^{0.00 \mid 0.00}$ (solid lines). Shown are differences between the TEOBResums model and different approximants (labeled with $X$ ) $\Delta Q_{\hat{\omega}}^{\mathrm{X}}=Q_{\hat{\omega}}^{\mathrm{TEOBResumS}}-Q_{\hat{\omega}}^{\mathrm{X}}$ rescaled by $\kappa_{\text {eff }}^{T}$. The inset shows $\Delta Q_{\hat{\omega}}^{\mathrm{X}}$ for lower frequencies. The purple markers correspond to frequencies $(50,100,200,400,800) \mathrm{Hz}$ for the $\mathrm{SLy}_{1.35 \mid 1.35}^{0.00 \mid 0.00}$ case.

the meaningful computation of differences with PN approximants in the $\mathrm{PN}$ regime $(\simeq 10-30 \mathrm{~Hz}$, see below). To overcome this difficulty, one has to properly downsample and smooth the raw output of TEOBResumS. Figure 9 shows together the $Q_{\hat{\omega}}$ computed for the three equal-mass binaries, one for BBHs, where tidal effects are set to zer ${ }^{9}$ (black, solid) and two BNSs, SLy $y_{1.35 \mid 1.35}^{0.00 \mid 0.00}$ (blue, dashed) and $\mathrm{H} 4_{1.37 \mid 1.37}^{0.00 \mid 0.00}$ (red, dash-dotted) up to $\hat{\omega} \approx 0.06$. The vertical lines mark $400 \mathrm{~Hz}$ and $700 \mathrm{~Hz}$ for $\mathrm{SLy}_{1.35 \mid 1.35}^{0.00 \mid 0.00}$ (blue) and $\mathrm{H} 4_{1.37 \mid 1.37}^{0.00 \mid 0.00}$ (red). The plot synthetically illustrates two things: (i) the effect of the tidal effects on phasing, that is attractive, is to push the curve down, (ii) the numerical value of the curve up to $\hat{\omega} \simeq 0.06$ is of order 100 . Since then $\epsilon_{\text {adiab }} \sim 0.01$, consistently with [69, we conclude that in this regime the SPA is still a valid approximation and as such it is meaningful to compare the so-computed time-domain $Q_{\hat{\omega}}$ with the corresponding ones obtained from the frequency domain approximants. Following precisely the same reasoning of 69, we extract the tidal part of $Q_{\hat{\omega}}$ from TEOBResumS, to compare it directly with the corresponding ones obtained from NRTidal, Eq. (6), or the PN-expanded one, Eq. (20). We computed the tidal part of the TEOBResumS $Q_{\hat{\omega}}$ as

$$
Q_{\hat{\omega}}^{T_{\text {TEOBResums }}} \equiv Q_{\hat{\omega}}-Q_{\hat{\omega}}^{0}
$$

where $Q_{\hat{\omega}}^{0}$ indicates the point-mass curve. We present our results in terms of differences between $Q_{\hat{\omega}}^{\mathrm{T} \text { TEOBResums }}$ and

\footnotetext{
9 This is obtained running TEOBResums without next-to-quasicircular correction to the waveform and flux 65], for consistency with the tidal part where these effects are not included at all.
} 
NRTidal, Eq. 6) or various PN truncations of Eq. 20, i.e., we define the quantity

$$
\Delta Q_{\hat{\omega}}^{X}=Q_{\hat{\omega}}^{T_{\text {TEOBResums }}}-Q_{\hat{\omega}}^{T_{X}}
$$

that is shown, rescaled by $\kappa_{\text {eff }}^{T}$, in Fig. 10 for $\mathrm{SLy}_{1.35 \mid 1.35}^{0.00 \mid 0.00}$ (dashed lines) and $\mathrm{H} 4_{1.37 \mid 1.37}^{0.00 \mid 0.00}$ (solid lines). On the same plot, the purple markers correspond to frequencies $(50,100,200,400,800) \mathrm{Hz}$ for $\mathrm{SLy}_{1.35 \mid 1.35}^{0.00 \mid 0.00}$. The plot illustrates the following facts.

(i) In the "early" frequency range $f \lesssim 150 \mathrm{~Hz}$ (see inset), for both configurations the difference between the NRTidal approximant and the TEOBResumS model is below $10^{-5}$ and always smaller than for the PN approximants. This keeps being small also after multiplication by $k_{\text {eff }}^{T} \approx 10^{2}$, which assures that the two models just negligibly dephase up to $150 \mathrm{~Hz}$. This confirms the quality of the calibration of the NRTidal model to TEOBResums, illustrating that the fit of the high-frequency part, probably thank to having imposed the correct LO tidal behavior, did not lead to large uncertainties at low frequencies. The same plot also shows that even at frequencies $\sim 50 \mathrm{~Hz}$, i.e., several hundred orbits before the actual merger there are noticeable differences between the tidal PN approximants and TEOBResums. This indicates that the $\mathrm{PN}$ regime is not yet met there (and it in fact extends below $30 \mathrm{~Hz}$ ) so that one should not, in principle, restrict to the use of simple PN-expanded descriptions of tidal effects. One sees in this respect that the $1 \mathrm{PN}$ tidal approximant overestimates the effects (i.e., the inspiral is accelerated), while all the other approximant underestimate them with respect to TEOBResums.

(ii) when moving to higher frequencies $f \gtrsim 150 \mathrm{~Hz}$, it is found that NRTidal yields stronger tidal effects with respect to the TEOBResums baseline. Once multiplied by $\kappa_{\text {eff }}^{T}$, the differences shown in the figure become $\sim 2$ for $\operatorname{SLy}_{1.35 \mid 1.35}^{0.00 \mid 0.00}$ and order $\sim 6$ for $\mathrm{H} 4_{1.37 \mid 1.37}^{0.00 \mid 0.00}$ at $700 \mathrm{~Hz}$, which implies an accumulated phase difference up to that frequency of the order of a radian. As a consequence when NRTidal is used to extract the tidal parameters from actual GW signals, one may expect to get smaller tidal deformabilities with respect to those predicted by PN or EOB approximants, see the recent study of [83, 105], in order to compensate for the aforementioned systematics in the post-LO tidal correction yielded by $P_{\Psi}^{\mathrm{NRTidal}}$, Eq. 6 .

(iii) The third piece of information yielded by Fig. (10) is that the dashed and solid lines do not coincide. This is not surprising, and actually expected, since $\kappa_{2}^{T}\left(\right.$ or $\left.\kappa_{\text {eff }}^{T}\right)$ only takes into account leading-order (conservative) tidal effects, while their incorporation in a state-of-the-art EOB model is more complicated [12, 14, 46, 49, 102. For example, the

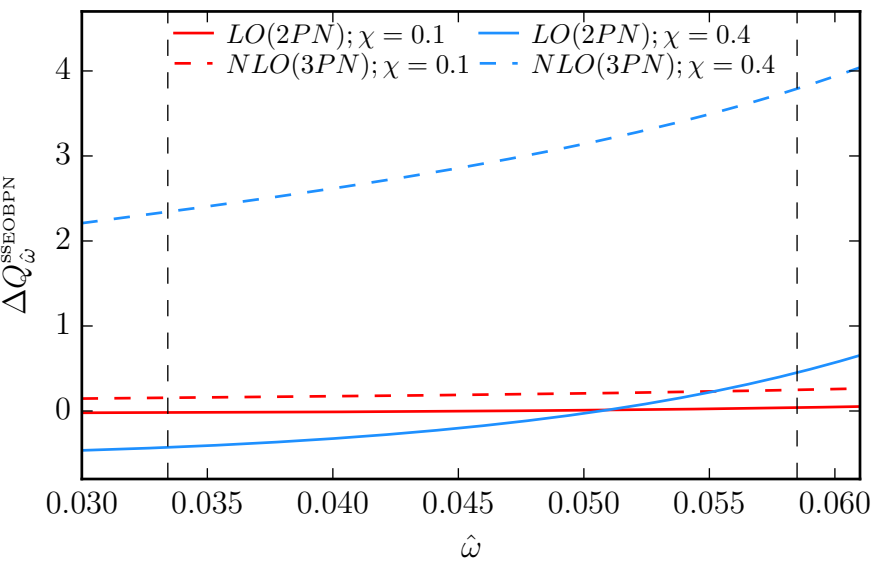

FIG. 11. $Q_{\hat{\omega}}$-description of the self-spin contribution to the phasing for an equal-mass binary with $M^{A}=M^{B}=1.35 \mathrm{em}$ ploying the MS1b EOS. The spin magnitudes are $\chi^{A}=\chi^{B}=$ 0.1 (red) and $\chi^{A}=\chi^{B}=0.4$ (blue). The vertical dashed lines refer to 400 and $700 \mathrm{~Hz}$. The plot shows the difference $\Delta Q_{\hat{\omega}}^{\mathrm{SS} \mathrm{EOBPN}}$ between the self-spin $Q_{\hat{\omega}}^{\mathrm{sS}}{ }^{\mathrm{SOB}}$ obtained with the TEOBResums model and the corresponding PN-expanded expressions at LO (2PN, solid lines [33]) or NLO (3PN, dashed lines 67]), as actually implemented in PhenomPv2_NRTidal. For astrophysically motivated values of the spins, 0.1 , the effect of the PN NLO term is still compatible with TEOBResums, that is constructed using only LO self-spin information in the Hamiltonian and in the flux. By contrast, it is not the case for larger values of the spins, so that a full assessment of the approximant in that regime calls for modification of the self-spin content of TEOBResums.

presence of $\ell=3$ and $\ell=4$ tidal corrections (that become more important the more deformable the star is) as well as of additional, mass-ratiodependent, effects [14, 102] that were not incorporated in the simplified (mass-ratio independent) fit given by $P_{\Psi}^{\text {NRTidal }}$ in Eq. (6). Still, as it should, the linearity in the tidal parameter is recovered correctly in the medium-low frequency regime 30$100 \mathrm{~Hz}$.

\section{Contributions due to the spin-induced quadrupole moment}

Similarly to the discussion about the tidal contribution to the GW phasing, we also want to discuss the imprint of the quadrupole-dependent spin-spin effects as they are implemented in PhenomPv2_NRTidal. As mentioned above, PhenomPv2_NRTidal, as well as TaylorF2 Tides, implement EOS-dependent self-spin terms up to NLO, i.e. that include both the LO, $2 \mathrm{PN}$-accurate, term 33 and as well as the $3 \mathrm{PN}$-accurate term that can be deduced from Ref. [67. By contrast, TEOBResums only implements self-spin information at LO in both 
the Hamiltonian and the flux 10 Since we used the TEOBResums model as baseline to validate the performance of PhenomPv2_NRTidal, we need to check that the effect of the NLO terms present in PhenomPv2_NRTidal is not dramatically stronger than the LO ones over the explored parameter range. To do so, we isolate the selfspin contribution, $Q_{\hat{\omega}}^{\text {ss }}$, to the phasing of TEOBResumS and compare it with the LO and NLO contributions in PhenomPv2_NRTidal. We follow the procedure discussed in Ref. [37, where $Q_{\hat{\omega}}^{\text {ss }}$ is extracted by subtracting from the total $Q_{\hat{\omega}}$ the corresponding one obtained putting the quadrupolar deformation parameters, $C_{Q}$, to zero. In order to get an upper limit to the effect, we chose and equal-mass configuration $M^{A}=M^{B}=1.35 M_{\odot}$ for the MS1b EOS, due to the large (unphysical) deformability, and we put either $\chi^{A}=\chi^{B}=0.1$ or $\chi^{A}=\chi^{B}=0.4$. The value of the quadrupolar parameter induced by rotation in this case is $C_{Q A}=C_{Q B} \simeq 8.396$. For the sake of comparison, we recall that $C_{Q A}=C_{Q B}=1$ for the $\mathrm{BH}$ case, and $C_{Q A}=C_{Q B} \simeq 5.49$ for a SLy configurations with the same individual masses. Figure 11 depicts the difference $\Delta Q_{\hat{\omega}}^{\mathrm{SS}}=Q_{\hat{\omega}}^{\mathrm{SSEOB}}-Q_{\hat{\omega}}^{\mathrm{SSPN}}$ for both the LO (solid) and NLO (dashed) PN truncations and for the two values of the spins. The two vertical lines refer to 400 and $700 \mathrm{~Hz}$. The figure illustrates several facts. Focusing first on the, astrophysical motivated, small spin case, one sees that both the LO and NLO truncations of the PN approximants are essentially consistent with TEOBResumS. More precisely, the EOB - PN phase difference accumulated between $20 \mathrm{~Hz}(\hat{\omega} \simeq 0.00251$, not represented in the figure) and $\hat{\omega}=0.06$ is $\Delta \phi_{\mathrm{LO}}^{\mathrm{ss}}=-0.063$ in the LO case and $\Delta \phi_{\mathrm{NLO}}^{\mathrm{sS}}=+0.348$ in the NLO case. More precisely, as shown in Fig. 14 of 37, one finds that the NLO term used in the PhenomPv2_NRTidal model overall increases the effect of the self-spin contribution such that it is globally stronger, and thus more attractive, than TEOBResumS. In any case, this analysis brings us to the conclusion that, for astrophysically motivated values of the spins, we can use TEOBResumS to validate PhenomPv2_NRTidal even if the NLO self-spin PN terms are not included in TEOBResums.

When the spins are as large as 0.4, a similar qualitative behavior is found, but the accumulated dephasings become larger, with $\Delta \phi_{\mathrm{LO}}^{\mathrm{ss}}=-1.29$ and $\Delta \phi_{\mathrm{NLO}}^{\mathrm{ss}}=+5.31$. Since the analytically known low-frequency NLO self-spin behavior is not incorporated in TEOBResumS, we cannot prove whether this difference is physical, due to the expected bad behavior of the PN series, or the missing NLO self-spin contribution in TEOBResums. To do so, we would need to upgrade TEOBResumS incorporating NLO self-spin information in the Hamiltonian, radiation reaction and waveform. This will be the subject of a forthcoming

10 We recall that, since the EOB waveform is resummed, other terms, notably the tail ones, are effectively included in the TEOBResums expression for the flux, while they are not part of the PN approximants. study. For the time being, however, let us note that we are prone to suspect that the large EOB/PN difference in Fig. 11 for spins 0.4 is (at least partly) due to the nonrobust behavior of the NLO PN approximant. The basis of our reasoning comes from inspecting the magnitude of each term of the PN expansion of the self-spin part of $Q_{\hat{\omega}}, Q_{\hat{\omega}}^{\mathrm{SS}}$. Following Ref. [37] (see Eqs. (51)-(53) there), the PN-expanded function reads

$$
Q_{\omega}^{\mathrm{PN}, \mathrm{SS}}=Q_{\omega}^{\mathrm{SS} \mathrm{PN}, \mathrm{LO}}+Q_{\omega}^{\mathrm{SS} \mathrm{PN}, \mathrm{NLO}}
$$

where

$$
\begin{aligned}
Q_{\omega}^{\mathrm{SS} \mathrm{PN}_{\mathrm{LO}} \mathrm{O}} & =-\frac{25}{48 \nu}\left(\tilde{a}_{A}^{2} C_{Q A}+\tilde{a}_{B}^{2} C_{Q B}\right)\left(\frac{\hat{\omega}}{2}\right)^{-1 / 3}, \\
Q_{\omega}^{\mathrm{SS} \mathrm{PN}, \mathrm{NLO}} & =-\frac{1}{\nu}\left[\left(\frac{5}{8} \nu+\frac{15635}{4032}\right)\left(C_{Q A} \tilde{a}_{A}^{2}+C_{Q B} \tilde{a}_{B}^{2}\right)\right. \\
& \left.+\frac{2215}{2304} X_{A B}\left(C_{Q A} \tilde{a}_{A}^{2}-C_{Q B} \tilde{a}_{B}^{2}\right)\right]\left(\frac{\hat{\omega}}{2}\right)^{1 / 3} .
\end{aligned}
$$

Evaluating the above equations for $\chi_{A}=\chi_{B}=+0.4$ at $\hat{\omega}=0.06$, one finds $Q_{\omega}^{\mathrm{SS}, \mathrm{LO}} \simeq-4.5$ while $Q_{\omega}^{\mathrm{SS} \text { PN,LO}} \simeq$ -3.37 . The fact that the magnitude of the NLO and LO terms are comparable suggests that one is in a regime (of spin and frequencies) where the reliability of the straightforward PN description is questionable.

On a positive side, one has to remember that the results of Fig. 11 refer to an EOS that has very reduced probability of exist in Nature, which seems to select softer EOS models with smaller rotation-induced quadrupole moment 11. This suggests that the effect of the NLO terms in PhenomPv2_NRTidal is subdominant with respect to the LO ones and the comparisons with the TEOBResumS baseline should be considered reliable.

\section{SUMMARY AND FUTURE PERSPECTIVES}

We described in detail the implementation of the NRTidal models in the LSC Algorithm Library Suite, along with detailed tests of these new waveform approximants. Our study is timely, as such approximants were already employed to estimate the properties of the source of the GW signal GW170817 and they will be employed for further analysis of the system. To validate the performance of the NRTidal models, we computed mismatches, frequency-domain phase differences, and timedomain phase differences between the different waveform models and target BNS waveforms (an approximation of the true GW signal) constructed by hybridizing tidally improved EOB waveforms, obtained via the TEOBResumS model [37, with high-resolution NR simulations covering the last orbits of the inspiral up to merger. The additional new theoretical input incorporated in TEOBResumS is that the model blends together, in a resummed fashion, 
tidal and spin effects, notably including EOS-dependent self-spin effects. This special feature not only allows one to assess the performance of NRTidal models, but also that of the PN-based description of the self-spin effects that is present in some of the LAL approximants.

Our main observations are:

(i) For spinning BNSs or stiff EOSs, non-tidal approximants fail to describe the evolution of the binary system. Consequently, every analysis discussing properties of BNS systems has to be based on waveform approximants incorporating tidal effects.

(ii) For spinning systems, the inclusion of the spininduced, and EOS-dependent 2PN term [33] (and $3 \mathrm{PN}$ [67]) terms in the waveform approximants proves crucial to reduce the mismatches with the TEOBResumS-driven inspiral waveform when the spin magnitudes are $\gtrsim 0.1[36$.

(iii) For configurations with large unequal masses $(q=$ 1.5) and/or large tidal effects, the performance of the NRTidal models is better than the performance of the PN based waveform approximants by more than an order of magnitude in terms of mismatches.

(iv) We compared and contrasted in detail the NRTidal representation of the tidal interaction with the one incorporated in TEOBResumS. We concluded that the NRTidal model systematically overestimates the tidal interaction with respect to TEOBResums also in the intermediate frequency $150-800 \mathrm{~Hz}$ where TEOBResums is expected to be reliable. If, on the one hand, this calls for improvements in the construction of NRTidal, on the other hand, despite the very small mismatches found $\left(<10^{-2}\right.$ of $\left.\sim 10^{-3}\right)$, one should be careful about possible systematics in parameter estimation studies brought by the use of NRTidal. Such uncertainties should be properly assessed by means of injection studies employing full parameter estimation pipelines 11 .

(v) Finally, by comparing the PhenomPv2_NRTidal model also to a precessing NR simulation we conclude that the model looks sufficiently mature to deliver a qualitatively and semi-quantitatively consistent representation of the last orbits of precessing BNS systems.

11 Recent studies published after the submission of this manuscript, Refs. 83, 105, 106], show with full parameter estimation that for GW170817 or BNS mergers with similar signal-to-noise ratio the NRTidal approximants do not introduce systematic biases. For higher signal-to-noise ratios $(\geq 80)$ also a recovery of the masses, and effective spin is possible, but there are systematic measurement biases in the tidal deformability, e.g., 83 105. This is in broad agreement with simple accuracy estimates relating the mismatch and the minimum signal-to-noise ratio, Eq. (18) of [107, see also 108. Based on our analysis, we find that for typical mismatches of $\sim 3 \times 10^{-3}$ a minimum signal-to-noise ratio of $\geq 60$ would be needed for a possible bias in parameter estimation.
Based on the chosen target waveform set, we find that the PhenomPv2_NRTidal model gives the smallest mismatches and phase differences in the frequency and time domain. It is currently the only frequency model implemented in the LALSuite which incorporates phase corrections based on NR and EOB tuned tidal effects, the spin induced EOS dependent quadrupole moment, and precession of the orbital angular momentum for non-aligned spins.

Although the current implementation of the NRTidal waveform approximant is a step towards an efficient modeling of tidal effects in BNS systems like GW170817, there are immediate tests and improvements that we want to outline here to steer future developments of the model. The three obvious checks are (i) tests of the performance of the PhenomPv2_NRTidal model for precessing systems for the entire inspiral, (ii) tests against different hybrid models based on other waveform models and NR simulations, and (iii) tests the effect of the waveform approximant in the context of parameter estimation studies. Considering (ii) and (iii), we remark that there are ongoing injection studies to assess systematic uncertainties of waveform approximants for parameter estimation purposes. Furthermore, the implementation of the tidal EOB model SEOBNRv4T [11, which includes the EOS dependent quadrupole effect, was recently completed. This will allow to construct additional hybrids using models that rely on different inspiral waveforms. However, there is currently no possibility to compare the PhenomPv2_NRTidal model against precessing systems throughout the entire frequency band accessible to advanced GW detectors. While the recent progress in NR allowed us to validate the performance of PhenomPv2_NRTidal during the last 15 orbits before merger, there is no tidal EOB model which incorporates precession effects to enable a study of the early inspiral.

Considering possible improvements of the NRTidal approximants, we plan in the near future to (i) incorporate analytical tidal corrections to the amplitude of the GW, (ii) include the EOS dependence of the spininduced quadrupole momentum in the PhenomD_NRTidal and SEOBNRv4_ROM_NRTidal waveform models, and most notably, (iii) try to incorporate analytical knowledge beyond next-to-leading order tidal contributions to the NRTidal approximant to further improve the performance of the model throughout the entire inspiral.

\section{ACKNOWLEDGMENTS}

We want to thank Nathan Johnson-McDaniel for his participation in the LIGO review process of the NRTidal models, in particular for his checks of the merger frequency and comparisons to BBH systems and NR waveforms. We also thank Tanja Hinderer for her work on the review of the PhenomPv2_NRTidal model and Francesco Messina for carefully cross-checking the implementation of the self-spin terms in the TaylorF2 $2_{\text {Tides }}$ model. We 
also thank Sergei Ossokine for helpful discussions about precessing compact binary systems. We thank Alessandra Buonanno, Nathan Johnson-McDaniel, and Noah Sennett for comments on the manuscript.

TD acknowledges support by the European Union's Horizon 2020 research and innovation program under grant agreement No 749145, BNSmergers. PK acknowledges support at Cornell from the Sherman Fairchild Foundation and NSF grants PHY-1306125 and AST-1333129. FP acknowledges support from Cardiff University Seedcorn Funding AH21101018. SB acknowledges support by the EU H2020 under ERC Starting Grant, no. BinGraSp714626. CM acknowledges support by the European Union's Horizon 2020 research and innovation program under grant agreement No 753115, ACFD. KWT, AS, TD and CVDB are supported by the research programme of the Netherlands Organisation for Scientific Research
(NWO). SK, FO and YS are supported by the Max Planck Society's Independent Research Group Grant. MH acknowledges support by the Swiss National Science Foundation (SNSF). FP acknowledges support by Science and Technology Facilities Council (STFC) grant ST/L000962/1 and European Research Council Consolidator Grant 647839. RD has been supported by the DFG Research Training Group 1523/2 "Quantum and Gravitational Fields" .

Computations of the numerical relativity waveforms have been performed performed on the supercomputer SuperMUC at the LRZ (Munich) under the project number pr48pu, the compute cluster Minerva of the MaxPlanck Institute for Gravitational Physics, and on the Hydra and Draco clusters of the Max Planck Computing and Data Facility.
[1] B. P. Abbott et al. (Virgo, LIGO Scientific), Phys. Rev. Lett. 119, 161101 (2017), arXiv:1710.05832 [gr-qc]

[2] B. P. Abbott et al. (Virgo, Fermi-GBM, INTEGRAL, LIGO Scientific), Astrophys. J. 848, L13 (2017), arXiv:1710.05834 [astro-ph.HE],

[3] Astrophys. J. 848, L12 (2017), arXiv:1710.05833 [astroph.HE].

[4] D. A. Coulter, R. J. Foley, C. D. Kilpatrick, M. R. Drout, A. L. Piro, B. J. Shappee, M. R. Siebert, J. D. Simon, N. Ulloa, D. Kasen, B. F. Madore, A. MurguiaBerthier, Y.-C. Pan, J. X. Prochaska, E. Ramirez-Ruiz, A. Rest, and C. Rojas-Bravo, Science 358, 1556 (2017), arXiv:1710.05452 [astro-ph.HE]

[5] B. P. Abbott et al. (LIGO Scientific, VINROUGE, Las Cumbres Observatory, DLT40, Virgo, 1M2H, MASTER), Nature (2017), 10.1038/nature24471. arXiv:1710.05835 [astro-ph.CO].

[6] D. Radice, A. Perego, and F. Zappa, (2017), arXiv:1711.03647 [astro-ph.HE].

[7] A. Bauswein, O. Just, N. Stergioulas, and H.-T. Janka, (2017), arXiv:1710.06843 [astro-ph.HE]

[8] B. P. Abbott et al. (Virgo, LIGO Scientific), Astrophys. J. 832, L21 (2016) arXiv:1607.07456 [astro-ph.HE].

[9] J. Veitch et al., Phys. Rev. D91, 042003 (2015), arXiv:1409.7215 [gr-qc]

[10] S. Bernuzzi, A. Nagar, T. Dietrich, and T. Damour, Phys.Rev.Lett. 114, 161103 (2015), arXiv:1412.4553 [gr-qc].

[11] T. Hinderer et al., Phys. Rev. Lett. 116, 181101 (2016) arXiv:1602.00599 [gr-qc]

[12] J. Steinhoff, T. Hinderer, A. Buonanno, and A. Taracchini, Phys. Rev. D94, 104028 (2016), arXiv:1608.01907 [gr-qc].

[13] A. Buonanno and T. Damour, Phys. Rev. D59, 084006 (1999), arXiv:gr-qc/9811091.

[14] T. Damour and A. Nagar, Phys. Rev. D81, 084016 (2010), arXiv:0911.5041 [gr-qc]

[15] K. Hotokezaka, K. Kyutoku, H. Okawa, and M. Shibata, Phys. Rev. D91, 064060 (2015), arXiv:1502.03457 [gr-qc].

[16] T. Dietrich and T. Hinderer, Phys. Rev. D95, 124006
(2017), arXiv:1702.02053 [gr-qc]

[17] B. D. Lackey, S. Bernuzzi, C. R. Galley, J. Meidam, and C. Van Den Broeck, Phys. Rev. D95, 104036 (2017), arXiv:1610.04742 [gr-qc]

[18] B. D. Lackey, K. Kyutoku, M. Shibata, P. R. Brady, and J. L. Friedman, Phys.Rev. D89, 043009 (2014), arXiv:1303.6298 [gr-qc],

[19] F. Pannarale, E. Berti, K. Kyutoku, and M. Shibata, Phys. Rev. D88, 084011 (2013), arXiv:1307.5111 [grqc]

[20] K. Barkett et al., Phys. Rev. D93, 044064 (2016), arXiv:1509.05782 [gr-qc]

[21] J. Lange et al., Phys. Rev. D96, 104041 (2017), arXiv:1705.09833 [gr-qc]

[22] S. Bernuzzi, A. Nagar, M. Thierfelder, and B. Brügmann, Phys.Rev. D86, 044030 (2012), arXiv:1205.3403 [gr-qc].

[23] M. Favata, Phys.Rev.Lett. 112, 101101 (2014) arXiv:1310.8288 [gr-qc],

[24] L. Wade, J. D. E. Creighton, E. Ochsner, B. D. Lackey, B. F. Farr, T. B. Littenberg, and V. Raymond, Phys. Rev. D89, 103012 (2014), arXiv:1402.5156 [gr-qc].

[25] K. Hotokezaka, K. Kyutoku, Y.-i. Sekiguchi, and M. Shibata, Phys. Rev. D93, 064082 (2016), arXiv:1603.01286 [gr-qc].

[26] T. Dietrich, S. Bernuzzi, and W. Tichy, Phys. Rev. D 96, 121501 (2017).

[27] https://wiki.ligo.org/DASWG/LALSuite

[28] A. Bohé, L. Shao, A. Taracchini, A. Buonanno, S. Babak, I. W. Harry, I. Hinder, S. Ossokine, M. Pürrer, V. Raymond, T. Chu, H. Fong, P. Kumar, H. P. Pfeiffer, M. Boyle, D. A. Hemberger, L. E. Kidder, G. Lovelace, M. A. Scheel, and B. Szilágyi, Phys. Rev. D 95, 044028 (2017)

[29] S. Husa, S. Khan, M. Hannam, M. Pürrer, F. Ohme, X. J. Forteza, and A. Bohé, Phys. Rev. D 93, 044006 (2016)

[30] S. Khan, S. Husa, M. Hannam, F. Ohme, M. Pürrer, X. J. Forteza, and A. Bohé, Phys. Rev. D 93, 044007 (2016).

[31] M. Hannam, P. Schmidt, A. Bohé, L. Haegel, S. Husa, 
F. Ohme, G. Pratten, and M. Pürrer, Phys. Rev. Lett. 113, 151101 (2014)

[32] K. Kawaguchi, K. Kiuchi, K. Kyutoku, Y. Sekiguchi, M. Shibata, and K. Taniguchi, (2018), arXiv:1802.06518 [gr-qc]

[33] E. Poisson, Phys. Rev. D 57, 5287 (1998).

[34] M. Agathos, J. Meidam, W. Del Pozzo, T. G. F. Li, M. Tompitak, J. Veitch, S. Vitale, and C. Van Den Broeck, Phys. Rev. D92, 023012 (2015), arXiv:1503.05405 [gr-qc]

[35] N. V. Krishnendu, K. G. Arun, and C. K. Mishra, Phys. Rev. Lett. 119, 091101 (2017), arXiv:1701.06318 [gr-qc]

[36] I. Harry and T. Hinderer, (2018), arXiv:1801.09972 [gr$\mathrm{qc}]$.

[37] A. Nagar et al., (2018), arXiv:1806.01772 [gr-qc]

[38] T. Damour, "Gravitational radiation and the motion of compact bodies, in Gravitational Radiation, edited by N. Deruelle and T. Piran," (North-Holland, Amsterdam, 1983, 1983) pp. 59-144.

[39] T. Hinderer, Astrophys. J. 677, 1216 (2008), arXiv:0711.2420 [astro-ph]

[40] T. Damour and A. Nagar, Phys. Rev. D80, 084035 (2009), arXiv:0906.0096 [gr-qc]

[41] T. Binnington and E. Poisson, Phys. Rev. D80, 084018 (2009), arXiv:0906.1366 [gr-qc]

[42] T. Dietrich, S. Bernuzzi, B. Bruegmann, and W. Tichy (2018) arXiv:1803.07965 [gr-qc]

[43] E. Poisson, Phys. Rev. D57, 5287 (1998), arXiv:grqc/9709032 [gr-qc]

[44] M. Levi and J. Steinhoff, JCAP 1412, 003 (2014), arXiv:1408.5762 [gr-qc].

[45] J. E. Vines and E. E. Flanagan, Phys. Rev. D88, 024046 (2013), arXiv:1009.4919 [gr-qc]

[46] J. Vines, E. E. Flanagan, and T. Hinderer, Phys. Rev. D83, 084051 (2011), arXiv:1101.1673 [gr-qc]

[47] T. Damour and A. Nagar, Phys.Rev. D90, 044018 (2014), arXiv:1406.6913 [gr-qc]

[48] A. Nagar, T. Damour, C. Reisswig, and D. Pollney, (2015), arXiv:1506.08457 [gr-qc]

[49] T. Damour, A. Nagar, and L. Villain, Phys.Rev. D85, 123007 (2012), arXiv:1203.4352 [gr-qc]

[50] L. E. Kidder, Phys. Rev. D52, 821 (1995), arXiv:grqc/9506022 [gr-qc]

[51] T. A. Apostolatos, C. Cutler, G. J. Sussman, and K. S. Thorne, Phys. Rev. D49, 6274 (1994)

[52] A. Buonanno, Y.-b. Chen, and M. Vallisneri, Phys. Rev. D67, 104025 (2003), [Erratum: Phys. Rev.D74,029904(2006)], arXiv:gr-qc/0211087 [gr-qc]

[53] P. Schmidt, M. Hannam, S. Husa, and P. Ajith, Phys. Rev. D84, 024046 (2011), arXiv:1012.2879 [gr-qc].

[54] P. Schmidt, M. Hannam, and S. Husa, Phys. Rev. D86, 104063 (2012), arXiv:1207.3088 [gr-qc]

[55] P. Schmidt, F. Ohme, and M. Hannam, Phys. Rev. D91, 024043 (2015), arXiv:1408.1810 [gr-qc]

[56] K. Yagi and N. Yunes, Physics Reports 681, 1 (2017) approximate Universal Relations for Neutron Stars and Quark Stars.

[57] S. Bernuzzi, A. Nagar, S. Balmelli, T. Dietrich, and M. Ujevic, Phys.Rev.Lett. 112, 201101 (2014), arXiv:1402.6244 [gr-qc].

[58] T. Dietrich, N. Moldenhauer, N. K. JohnsonMcDaniel, S. Bernuzzi, C. M. Markakis, B. Brügmann, and W. Tichy, Phys. Rev. D92, 124007 (2015), arXiv:1507.07100 [gr-qc].
[59] T. Dietrich, M. Ujevic, W. Tichy, S. Bernuzzi, and B. Brügmann, Phys. Rev. D95, 024029 (2017), arXiv:1607.06636 [gr-qc]

[60] J. Healy, C. O. Lousto, and Y. Zlochower, Phys. Rev. D96, 024031 (2017), arXiv:1705.07034 [gr-qc]

[61] D. J. A. McKechan, C. Robinson, and B. S. Sathyaprakash, Gravitational waves. Proceedings, 8th Edoardo Amaldi Conference, Amaldi 8, New York, USA, June 22-26, 2009, Class. Quant. Grav. 27, 084020 (2010), arXiv:1003.2939 [gr-qc].

[62] J. S. Read, B. D. Lackey, B. J. Owen, and J. L. Friedman, Phys. Rev. D79, 124032 (2009), arXiv:0812.2163 [astro-ph]

[63] S. Bernuzzi, A. Nagar, and A. Zenginoglu, Phys. Rev. D86, 104038 (2012), arXiv:1207.0769 [gr-qc]

[64] E. Harms, S. Bernuzzi, A. Nagar, and A. Zenginoglu, Class. Quant. Grav. 31, 245004 (2014), arXiv:1406.5983 [gr-qc]

[65] A. Nagar, G. Riemenschneider, and G. Pratten, Phys. Rev. D96, 084045 (2017), arXiv:1703.06814 [gr-qc].

[66] F. Messina, A. Maldarella, and A. Nagar, (2018), arXiv:1801.02366 [gr-qc].

[67] A. Bohé, G. Faye, S. Marsat, and E. K. Porter, Class. Quant. Grav. 32, 195010 (2015), arXiv:1501.01529 [grqc]

[68] D. Bini, T. Damour, and A. Nagar, in prep. (2018).

[69] T. Damour, A. Nagar, and S. Bernuzzi, Phys. Rev. D87, 084035 (2013), arXiv:1212.4357 [gr-qc]

[70] B. Brügmann, J. A. Gonzalez, M. Hannam, S. Husa, U. Sperhake, et al., Phys.Rev. D77, 024027 (2008), arXiv:gr-qc/0610128 [gr-qc].

[71] M. Thierfelder, S. Bernuzzi, and B. Brügmann, Phys.Rev. D84, 044012 (2011), arXiv:1104.4751 [gr-qc]

[72] T. Dietrich, S. Bernuzzi, M. Ujevic, and B. Brügmann, Phys. Rev. D91, 124041 (2015), arXiv:1504.01266 [grqc]

[73] S. Bernuzzi and T. Dietrich, Phys. Rev. D94, 064062 (2016), arXiv:1604.07999 [gr-qc]

[74] S. Bernuzzi and D. Hilditch, Phys. Rev. D81, 084003 (2010), arXiv:0912.2920 [gr-qc]

[75] D. Hilditch, S. Bernuzzi, M. Thierfelder, Z. Cao, W. Tichy, et al., Phys. Rev. D88, 084057 (2013), arXiv:1212.2901 [gr-qc].

[76] C. Bona, J. Massó, J. Stela, and E. Seidel, in The Seventh Marcel Grossmann Meeting: On Recent Developments in Theoretical and Experimental General Relativity, Gravitation, and Relativistic Field Theories, edited by R. T. Jantzen, G. M. Keiser, and R. Ruffini (World Scientific, Singapore, 1996).

[77] M. Alcubierre, B. Brügmann, P. Diener, M. Koppitz, D. Pollney, et al., Phys.Rev. D67, 084023 (2003), arXiv:gr-qc/0206072 [gr-qc]

[78] J. R. van Meter, J. G. Baker, M. Koppitz, and D.I. Choi, Phys. Rev. D73, 124011 (2006), arXiv:gr$\mathrm{qc} / 0605030$

[79] M. Campanelli, C. O. Lousto, P. Marronetti, and Y. Zlochower, Phys. Rev. Lett. 96, 111101 (2006), arXiv:gr-qc/0511048

[80] J. G. Baker, J. Centrella, D.-I. Choi, M. Koppitz, and J. van Meter, Phys. Rev. Lett. 96, 111102 (2006) arXiv:gr-qc/0511103

[81] M. Dominik, K. Belczynski, C. Fryer, D. Holz, E. Berti, T. Bulik, I. Mandel, and R. O'Shaughnessy, Astrophys. J. 759, 52 (2012), arXiv:1202.4901 [astro-ph.HE] 
[82] J. M. Lattimer, Annual Review of Nuclear and Particle Science 62, 485 (2012), arXiv:1305.3510 [nucl-th].

[83] R. Dudi, F. Pannarale, T. Dietrich, M. Hannam, S. Bernuzzi, F. Ohme, and B. Bruegmann, (2018), arXiv:1808.09749 [gr-qc]

[84] A. Buonanno, B. R. Iyer, E. Ochsner, Y. Pan, and B. S. Sathyaprakash, Phys. Rev. D 80, 084043 (2009).

[85] A. Bohé, S. Marsat, and L. Blanchet, Classical and Quantum Gravity 30, 135009 (2013)

[86] A. Bohé, S. Marsat, and L. Blanchet, Class. Quant. Grav. 30, 135009 (2013), arXiv:1303.7412 [gr-qc]

[87] K. G. Arun, A. Buonanno, G. Faye, and E. Ochsner, Phys. Rev. D 79, 104023 (2009)

[88] B. Mikóczi, M. Vasúth, and L. A. Gergely, Phys. Rev. D 71, 124043 (2005).

[89] C. K. Mishra, A. Kela, K. G. Arun, and G. Faye, Phys. Rev. D93, 084054 (2016), arXiv:1601.05588 [gr-qc].

[90] M. Pürrer, Class. Quant. Grav. 31, 195010 (2014), arXiv:1402.4146 [gr-qc]

[91] "LIGO Document T0900288-v3," Advanced LIGO anticipated sensitivity curves.

[92] T. Damour, B. R. Iyer, and B. S. Sathyaprakash, Phys. Rev. D57, 885 (1998), arXiv:gr-qc/9708034 [gr-qc].

[93] T. Damour, B. R. Iyer, and B. S. Sathyaprakash, Phys. Rev. D62, 084036 (2000), arXiv:gr-qc/0001023 [gr-qc],

[94] T. Damour, B. R. Iyer, and B. S. Sathyaprakash, Phys. Rev. D63, 044023 (2001) [Erratum: Phys. Rev.D72,029902(2005)], arXiv:gr-qc/0010009 [gr-qc].

[95] A. Bohé, G. Faye, S. Marsat, and E. K. Porter, Classical and Quantum Gravity 32, 195010 (2015)

[96] M. Boyle, D. A. Brown, L. E. Kidder, A. H. Mroue, H. P.
Pfeiffer, M. A. Scheel, G. B. Cook, and S. A. Teukolsky, Phys. Rev. D76, 124038 (2007), arXiv:0710.0158 [grqc]

[97] T. Damour and A. Nagar, Phys. Rev. D77, 024043 (2008), arXiv:0711.2628 [gr-qc]

[98] L. Baiotti, T. Damour, B. Giacomazzo, A. Nagar, and L. Rezzolla, Phys. Rev. Lett. 105, 261101 (2010) arXiv:1009.0521 [gr-qc]

[99] T. Dietrich, S. Bernuzzi, B. Bruegmann, M. Ujevic, and W. Tichy, (2017), arXiv:1712.02992 [gr-qc].

[100] R. Haas et al., Phys. Rev. D93, 124062 (2016), arXiv:1604.00782 [gr-qc]

[101] K. Kiuchi, K. Kawaguchi, K. Kyutoku, Y. Sekiguchi, M. Shibata, and K. Taniguchi, Phys. Rev. D96, 084060 (2017), arXiv:1708.08926 [astro-ph.HE]

[102] D. Bini, T. Damour, and G. Faye, Phys. Rev. D85, 124034 (2012), arXiv:1202.3565 [gr-qc].

[103] T. Damour and A. Nagar, Phys. Rev. D76, 064028 (2007), arXiv:0705.2519 [gr-qc].

[104] D. Bini and T. Damour, Phys.Rev. D90, 124037 (2014), arXiv:1409.6933 [gr-qc],

[105] A. Samajdar and T. Dietrich, (2018), arXiv:1810.03936 [gr-qc]

[106] B. P. Abbott et al. (Virgo, LIGO Scientific), (2018), arXiv:1805.11579 [gr-qc]

[107] E. Baird, S. Fairhurst, M. Hannam, and P. Murphy, Phys. Rev. D87, 024035 (2013), arXiv:1211.0546 [grqc]

[108] C. Cutler and E. E. Flanagan, Phys. Rev. D49, 2658 (1994), arXiv:gr-qc/9402014 [gr-qc]. 